\title{
Maximum Induced Forests in Graphs of Bounded Treewidth
}

\author{
Glenn G. Chappell \\ Department of Computer Science \\ University of Alaska \\ Fairbanks, AK 99775-6670, USA \\ chappellg@member.ams.org
}

\author{
Michael J. Pelsmajer \\ Department of Applied Mathematics \\ Illinois Institute of Technology \\ Chicago, IL 60616, USA \\ pelsmajer@iit.edu
}

Submitted: Mar 18, 2011; Accepted: Oct 19, 2013; Published: Oct 28, 2013

2010 Mathematics Subject Classifications: Primary 05C35, Secondary 05C05

\begin{abstract}
Given a nonnegative integer $d$ and a graph $G$, let $f_{d}(G)$ be the maximum order of an induced forest in $G$ having maximum degree at most $d$. We seek lower bounds for $f_{d}(G)$ based on the order and treewidth of $G$.

We show that, for all $k, d \geqslant 2$ and $n \geqslant 1$, if $G$ is a graph with order $n$ and treewidth at most $k$, then $f_{d}(G) \geqslant\lceil(2 d n+2) /(k d+d+1)\rceil$, unless $G \in$ $\left\{K_{1,1,3}, K_{2,3}\right\}$ and $k=d=2$. We give examples that show that this bound is tight to within 1.

We conjecture a bound for $d=1: f_{1}(G) \geqslant\lceil 2 n /(k+2)\rceil$, which would also be tight to within 1 , and we prove it for $k \leqslant 3$. For $k \geqslant 4$ the conjecture remains open, and we prove a weaker bound: $f_{1}(G) \geqslant(2 n+2) /(2 k+3)$. We also examine the cases $d=0$ and $k=0,1$.

Lastly, we consider open problems relating to $f_{d}$ for graphs on a given surface, rather than graphs of bounded treewidth.
\end{abstract}

Keywords: Treewidth; chordal graph; induced forest

\section{Introduction}

We begin with an overview of the topics considered in this paper. Our terminology and notation agree with West [26]; see Diestel [13] for basic information about treewidth.

\section{$1.1 \quad$ Background}

Consider the following extremal problem for $\mathcal{G}$ a class of graphs. Given nonnegative integers $n, d$, find the greatest integer $t$ such that every $n$-vertex graph $G \in \mathcal{G}$ contains a $t$-vertex induced forest of maximum degree at most $d$. 
When $d=0$, we seek a lower bound on the size of a maximum independent set. This has been studied intensely for various graph classes $[3,14,17,19,20,25]$, and is the extremal version of the optimization problem Maximum Independent SeT. When $d=$ $n$, the desired forest has unrestricted degree. A vertex set $S$ in a graph $G$ is a minimumsize feedback vertex set if and only if the set $U=V(G)-S$ induces a forest of maximum order; thus the $d=n$ case is essentially an extremal version of FEEDBACK VERTEX SET. This topic has also received a great deal of study $[2,4,5,6,11,18,24]$. There are straightforward connections between these ideas and coloring and acyclic coloring (the latter were explored by Fertin et al. [15]), which can be used to obtain results for the $d=0$ and $d=n$ cases, respectively.

Most of the graph classes considered in the above papers are defined by planarity, degree restrictions, and girth restrictions. For the classes of planar graphs and outerplanar graphs, the first author considered the $d=2$ case, conjecturing lower bounds and giving examples that would be best possible. The second author proved the outerplanar graph conjecture [21, Thm. 1], showing that each $n$-vertex outerplanar graph contains an induced forest with at least $(4 n+2) / 7$ vertices and maximum degree at most 2 ; this is best possible.

In the present paper, we extend this result, considering the class of all graphs with treewidth at most some integer $k$. This is a natural outgrowth of the previous work, since outerplanar graphs have treewidth at most 2. We generalize a technique of Pelsmajer [21], to obtain nearly sharp results.

\subsection{Basic Definitions}

All graphs in this paper are finite, simple, and undirected.

If $G$ is a graph, then $V(G)$ denotes the vertex set of $G$. If $U \subseteq V(G)$, then $G[U]$ is the subgraph induced by the vertex set $U$. The set of all vertices adjacent to a vertex $v$ is the neighborhood of $v$, denoted by $N(v)$.

A graph $G$ is chordal if every cycle in $G$ of length at least 4 has a chord (an edge joining nonconsecutive vertices in the cycle). A chordal graph with at least one vertex must contain a vertex that is simplicial, that is, whose neighborhood induces a complete subgraph. The treewidth of a graph $G$ is equal to one less than the smallest possible clique number of a chordal graph of which $G$ is a subgraph.

A special kind of chordal graph is a $k$-tree, inductively defined as follows: given a nonnegative integer $k$, the complete graph $K_{k+1}$ is a $k$-tree. If $G$ is a $k$-tree, then the graph obtained by adding a new vertex and joining it to every vertex of some $k$-clique of $G$, is also a $k$-tree. Equivalently, a graph $G$ is a $k$-tree if $G$ has treewidth $k$ and is edge-maximal with respect to that property. Two well known facts about $k$-trees that we will use are that a $k$-tree cannot be disconnected by the removal of fewer than $k$ vertices, and that a $k$-tree cannot contain a subdivision of the complete graph $K_{k+2}$. (For more on $k$-trees and treewidth, see Diestel [13] or other references [7, 23].)

Suppose we assign numerical weights to the vertices of a graph $G$. The weight of $G$, denoted by $w(G)$, is the sum of the weights of the vertices of $G$. The weight of a set $U \subseteq V(G)$, denoted by $w(U)$, is the sum of the weights of the elements of $U$. 
If $S$ is a set, then we abbreviate $S \cup\{x\}$ by $S+x$, and we abbreviate $S-\{x\}$ by $S-x$.

\subsection{Discussion of Results}

The results in this paper concern a graph parameter that we denote by $f_{d}(G)$. Given a graph $G$, for each nonnegative integer $d$ we define $f_{d}(G)$ to be the maximum order of an induced forest in $G$ having maximum degree at most $d$.

For nonnegative integers $k, d$, we define a real number

$$
r_{k, d}:=\frac{2 d}{k d+d+1}
$$

As we will see, for graphs with treewidth $k$ and order $n$, the best lower bound for $f_{d}(G)$ is often something like $r_{k, d} \cdot n$.

The primary goal of this paper is to prove the following result.

Theorem 1. Let $k, d$ be integers, with $k \geqslant 2$ and $d \geqslant 2$. If $G$ is a graph with order $n \geqslant 1$ and treewidth at most $k$, then

$$
f_{d}(G) \geqslant\left\lceil\frac{2 d n+2}{k d+d+1}\right\rceil=\left\lceil r_{k, d} \cdot n+\frac{r_{k, d}}{d}\right\rceil,
$$

unless $G \in\left\{K_{1,1,3}, K_{2,3}\right\}$ and $k=d=2$.

The special case of Theorem 1 when $G$ is outerplanar and $d=2$ was proven by Pelsmajer [21, Thm. 1].

If a chordal graph has clique number $\omega$, then its treewidth is $\omega-1$. Further, $K_{2,3}$ is not chordal, and so Theorem 1 has the following corollary (which easily implies Theorem 1, as well).

Corollary 2. Let $d \geqslant 2$. If $G$ is a chordal graph with order $n$ and clique number $\omega \geqslant 3$, then

$$
f_{d}(G) \geqslant\left\lceil\frac{2 d n+2}{\omega d+1}\right\rceil
$$

unless $G \cong K_{1,1,3}$ and $d=2$.

In Section 2 we prove Theorem 1.

In Section 3 we discuss the sharpness of Theorem 1. We construct examples to show that, for each $k, d \geqslant 2$, the bound of Theorem 1 is tight to within 1 for all $n \geqslant 1$, and exactly sharp for infinitely many values of $n$. We conclude that the coefficient of $n$ in Theorem 1 (i.e., $r_{k, d}$ ) cannot be improved.

In Section 4 we discuss similar questions for $k<2$ and for $d<2$. We observe that the bound of Theorem 1 does not hold when $d=1$. We conjecture that in this case a slightly weaker bound holds, with no " +2 " in the numerator. 
Conjecture 3. Let $k \geqslant 0$. If $G$ is a graph with order $n$ and treewidth at most $k$, then

$$
f_{1}(G) \geqslant\left\lceil\frac{2 n}{k+2}\right\rceil=\left\lceil r_{k, 1} \cdot n\right\rceil .
$$

We show that, as with Theorem 1, the above bound is tight to within 1, if it holds.

The question of whether we can place " +2 " in the numerator may seem minor. However, it has a significant impact on how we handle questions about sharpness. If there is no extra constant in the numerator, then our bound is simply a fraction of $n$, as in Conjecture 3 , and we can reasonably expect to prove sharpness using a single graph as an example; larger examples can be constructed using disjoint unions of copies of this graph. However, if there is a constant in the numerator, as in Theorem 1, then we will need to construct an infinite family of graphs in order to prove sharpness.

We prove Conjecture 3 for $k \leqslant 3$. For $k \geqslant 4$, we prove a weaker bound: $f_{1}(G) \geqslant$ $(2 n+2) /(2 k+3)$. We also cover the cases $d=0$ and $k=0,1$, and we discuss sharpness.

In Section 5, we discuss related conjectures and results for planar graphs and graphs on other surfaces, including the following conjecture.

Conjecture 4. Let $d \geqslant 2$ be an integer. If $G$ is a planar graph with order $n \geqslant 1$, then

$$
f_{d}(G)>\frac{2 d n}{4 d+1}=r_{3, d} \cdot n \text {. }
$$

\subsection{Related Work}

We conclude Section 1 with a discussion of a related paper.

Bose, Dujmović, and Wood [10] considered a problem similar to ours. They examine a graph parameter that we will denote by $\hat{f}_{d}(G)$ : the maximum order of an induced forest in $G$ whose vertices all have degree at most $d$ in the original graph. Equivalently, they first discard all vertices of $G$ with degree greater than $d$, and then find the maximum order of an induced forest in the graph that remains. Like us, they focus on graphs with given order, whose treewidth is at most some integer $k$. For each triple $k, d, n$ with $k \geqslant 1$, $d \geqslant 2 k-1$, and $n \geqslant 2 k+1$, they prove the following bound:

$$
\hat{f}_{d}(G) \geqslant\left(\frac{d-2 k+1}{d-\frac{3}{2} k+1+\frac{1}{k+1}}\right)\left(\frac{2}{k+1}\right) n+\frac{2 k}{d-\frac{3}{2} k+1+\frac{1}{k+1}},
$$

for $G$ a graph with order $n$ and treewidth at most $k$.

Clearly, $\hat{f}_{d}(G) \leqslant f_{d}(G)$. Further, if $k$ and $n$ are fixed and $d$ increases without bound, then the best lower bounds for $f_{d}$ and $\hat{f}_{d}$ both approach $2 n /(k+1)$. On the other hand, for fixed $k, d$ with $d \leqslant 2 k-1$, the best lower bound for $\hat{f}_{d}$ is given by a constant [10, notes after Thm. 5.1], while $f_{d}$ grows with $n$ for all values of $k, d$. It is thus unsurprising that proving our results requires rather different methods.

The main result of Bose et al. [10, Thm. 5.1] is actually more general: they seek large induced subgraphs of treewidth at most some given $t$. Above we compared our result with the special case of theirs when $t=1$. We ask whether our result can be similarly extended to subgraphs of given treewidth. 
Problem 5. Generalize Theorem 1 to include lower bounds for the order of induced subgraphs of given treewidth.

\section{Proof of Theorem 1}

Rather than prove Theorem 1 directly, we use a loaded induction involving partial vertices (introduced by Pelsmajer [21]). Partial vertices are vertices of degree at most 1 that are required to be in the induced forest. When determining whether the induced forest is large enough, partial vertices in the forest count for less than other vertices. We denote the set of partial vertices by $P$.

The actual result we will prove is the following; Theorem 1 follows by setting $P=\varnothing$.

Lemma 6. Let $k, d$ be integers, with $k \geqslant 2$ and $d \geqslant 2$. Let $G$ be a graph with order $n \geqslant 1$ and treewidth at most $k$, and let $P \subseteq V(G)$ be an independent set, so that each vertex in $P$ has degree at most 1 in $G$. Let each vertex of $P$ have weight

$$
p:=\frac{2}{k d-d+1}
$$

and let each other vertex of $G$ have weight 1 . Then there exists a set $U$, with $P \subseteq U \subseteq$ $V(G)$, such that $G[U]$ is a forest with maximum degree at most $d$, and

$$
w(U) \geqslant \frac{2 d \cdot w(G)+2}{k d+d+1}=r_{k, d} \cdot w(G)+\frac{r_{k, d}}{d},
$$

unless $G \in\left\{K_{1,1,3}, K_{2,3}\right\}, P=\varnothing$, and $k=d=2$.

Note that $K_{1,1,3}$ and $K_{2,3}$ contain independent sets of size 3 , as well as other sets of 3 vertices that induce forests of maximum degree at most 2. If $G \in\left\{K_{1,1,3}, K_{2,3}\right\}$ and $k=d=2$, then $3=\frac{2 d \cdot w(G)+1}{k d+d+1}$.

Proof of Lemma 6 . Let $k, d, G, n, P, p$, and the vertex weights be as in the statement of the lemma. A vertex of weight $p$ is a partial vertex; other vertices are full. We proceed by induction on $w(G)$; this is valid because $w(G)$ can always be written as a rational number with denominator $k d-d+1$.

We may assume that $V(G-P)$ is nonempty, since otherwise $U=P=V(G)$ suffices, with $w(U)$ being exactly the required minimum when $|P|=1$, and strictly greater otherwise.

We may also assume that $G-P$ is either a $k$-tree or a complete graph with order at most $k+1$. If it is not, then we can add edges to $G-P$, without increasing its treewidth beyond $k$, until it lies in one of these categories. A set $U$ that meets the requirements for this modified graph will meet the requirements for the original graph, unless $k=d=2$, the modified graph lies in $\left\{K_{1,1,3}, K_{2,3}\right\}$, and the original graph does not. However, if the original graph is a 5 -vertex graph of treewidth at most 2 , other than $K_{1,1,3}$ or $K_{2,3}$, then 
there is always a way to add edges so that the modified graph is a 2-tree other than $K_{1,1,3}$ (or $K_{2,3}$, which is not a 2-tree).

We may further assume that each partial vertex has degree equal to 1 ; otherwise, modify $G$ by adding an edge from each isolated partial vertex to an arbitrary vertex of $G-P$. Any solution for the modified graph will suffice for the original graph.

Since $G-P$ is connected, each partial vertex has a neighbor, and the partial vertices form an independent set, it follows that $G$ is connected.

How Our Argument Works - We will break the argument into cases. In all cases of the proof except the "Base Cases", we define a graph $G^{\prime}$ based on $G$. We do this by removing vertices from $G$ and/or making full vertices partial; sometimes we also add new partial vertices. This $G^{\prime}$ will always have order at least 1 , treewidth at most $k$, and weight strictly less than $w(G)$. Where possible, we apply the induction hypothesis to $G^{\prime}$ to find a vertex set $U^{\prime}$ that is a solution for $G^{\prime}$. We then use this $U^{\prime}$ to construct the required vertex set $U$.

We cannot apply the induction hypothesis to $G$ if $k=d=2$ and $G^{\prime} \in\left\{K_{1,1,3}, K_{2,3}\right\}$. If we know that $G^{\prime}$ contains at least one partial vertex, then this is not a problem; however, in some cases, there is the possibility of $G^{\prime}$ having no partial vertices. ${ }^{1}$ When $G^{\prime} \in\left\{K_{1,1,3}, K_{2,3}\right\}$ we may simply specify $U$ directly. Another option is to set $U^{\prime}$ to be a 3-vertex set in $G^{\prime}$, and then construct $U$ based on $U^{\prime}$, as before. We will discuss this option further shortly.

Having constructed $U$, we need to show that it induces a forest in $G$ meeting the degree requirement (maximum degree at most $d$ ) and the weight requirement $(w(U)$ at least the required minimum). Usually, the former will follow clearly from the construction of $U$ based on $U^{\prime}$ and the fact that $G^{\prime}\left[U^{\prime}\right]$ is a forest of maximum degree at most $d$. We consider the latter.

Showing That $U$ Satisfies the Weight Requirement-In most cases, we obtain $U^{\prime}$ in $G^{\prime}$ by applying the induction hypothesis, and then we define $U$ based on $U^{\prime}$. To verify that $U$ has the required weight, note that it suffices to show that

$$
\frac{w(U)-w\left(U^{\prime}\right)}{w(G)-w\left(G^{\prime}\right)} \geqslant r_{k, d}
$$

In a case where $G^{\prime} \in\left\{K_{1,1,3}, K_{2,3}\right\}$ is a possibility and we define $U$ based on $U^{\prime}$, we can prove that $U$ satisfies the weight requirement by verifying the following strict weight inequality:

$$
\frac{w(U)-w\left(U^{\prime}\right)}{w(G)-w\left(G^{\prime}\right)}>r_{k, d}
$$

To see that this suffices, observe that $w\left(U^{\prime}\right) \geqslant\left(2 d \cdot w\left(G^{\prime}\right)+1\right) /(k d+d+1)$ [note the " +1 " in the numerator] when $U^{\prime}$ is obtained by applying the induction hypothesis and

\footnotetext{
${ }^{1}$ There are six cases later in the proof in which it is possible both that $G^{\prime}$ contains no partial vertices and that $k=d=2$ : those labeled Reductions A \& B, C2, C8, C12, and C14.
} 
also when $G^{\prime} \in\left\{K_{1,1,3}, K_{2,3}\right\}$ and $\left|U^{\prime}\right|=3$. Now rewrite the strict weight inequality:

$$
\begin{aligned}
w(U) & >w\left(U^{\prime}\right)+r_{k, d} \cdot\left[w(G)-w\left(G^{\prime}\right)\right] \\
& \geqslant \frac{2 d \cdot w\left(G^{\prime}\right)+1}{k d+d+1}+\frac{2 d \cdot\left[w(G)-w\left(G^{\prime}\right)\right]}{k d+d+1}=\frac{2 d \cdot w(G)+1}{k d+d+1} .
\end{aligned}
$$

Since the first inequality above is strict, and all numerators and denominators are integers, $w(U)$ must be at least $(2 d \cdot w(G)+2) /(k d+d+1)["+2 "$ in the numerator], as desired.

Notes on $K_{1,1,3}$ and $K_{2,3}$ - In the subsequent argument, whenever $k=d=2$ and $G^{\prime}$ has 5 vertices and no partial vertices, $G^{\prime}$ is always obtained by removing some vertices from $G$. Since $G-P$ is a $k$-tree or a complete graph, it follows that $G$ is chordal. Since chordal graphs are closed under taking induced subgraphs, $G^{\prime}$ will be chordal as well. The graph $K_{2,3}$ is not chordal, and so $G^{\prime} ¥ K_{2,3}$. Thus, in the remainder of this proof, the only special case for $G^{\prime}$ is $K_{1,1,3}$; we will never need to deal directly with $K_{2,3}$.

We will sometimes apply the following reasoning when dealing with $G^{\prime} \cong K_{1,1,3}$.

Remark 7 . When $k=d=2, G^{\prime} \cong K_{1,1,3}$, and $G^{\prime}$ is created by removing a connected subgraph $B$ from $G$, it may be helpful to let $U^{\prime}$ be a 3 -vertex set in $G^{\prime}$ that contains no vertices adjacent to vertices in $B$.

To see that we can always do this, let $N(B)$ be the vertices of $G^{\prime}$ that are adjacent to vertices in $B$. If $N(B)$ contains 2 nonadjacent vertices or $N(B)$ contains a triangle, then, since $B$ is connected and $G^{\prime} \cong K_{1,1,3}$, there is a subdivision of $K_{4}$ in $G$. But this is impossible, since a graph of treewidth at most 2 cannot contain a subdivision of $K_{4}$. Therefore, $N(B)$ contains either exactly one vertex or two adjacent vertices of $G^{\prime}$. Considering all the possibilities, we can see that there is always a 3 -vertex set $U^{\prime}$ in $G^{\prime}-N(B)$ that does not form a triangle, or, equivalently, induces a forest of maximum degree at most 2 .

\subsection{Simple Base Cases and Reductions}

Base Case 1-Suppose that $G-P$ has exactly one vertex, $v$.

If $v$ has more than $d$ (partial) neighbors, then let $U=V(G)-v$. This $U$ is an independent set. Since $w(G)=w(U)+1$, the desired bound becomes

$$
w(U) \geqslant \frac{2 d[w(U)+1]+2}{k d+d+1}
$$

which simplifies to

$$
w(U) \geqslant \frac{2(d+1)}{k d-d+1}=(d+1) p .
$$

Because $U$ has at least this weight, the weight requirement is satisfied.

Otherwise, $G$ is a tree with maximum degree at most $d$. Let $U=V(G)$, so that $w(G)=w(U)$. The desired bound becomes

$$
w(U) \geqslant \frac{2 d \cdot w(U)+2}{k d+d+1},
$$


which simplifies to

$$
w(U) \geqslant \frac{2}{k d-d+1}=p,
$$

and again the weight requirement is satisfied.

Henceforth, we assume that $G-P$ has at least two vertices.

Reduction $\mathbf{A}$-Suppose that a full vertex $v$ of $G$ has $d$ or more partial neighbors. (See the first graph in Figure 1.)

Remove $v$ and its partial neighbors, and let $G^{\prime}$ be the graph that remains.

First suppose that $G^{\prime} \neq K_{1,1,3}$ or $k>2$ or $d>2$. There is at least one full vertex in $G^{\prime}$ (otherwise $G$ is Base Case 1), and so we find $U^{\prime}$ in $G^{\prime}$ by the induction hypothesis. Let $U$ be $U^{\prime}$ together with the removed partial vertices. Then $G[U]$ is $G^{\prime}\left[U^{\prime}\right]$ plus isolated vertices, and so $G[U]$ is a forest satisfying the degree requirement. We have

$$
\begin{array}{rlr}
\frac{w(U)-w\left(U^{\prime}\right)}{w(G)-w\left(G^{\prime}\right)} & \geqslant \frac{d p}{1+d p} \\
& =\frac{2 d}{k d+d+1} \quad \text { set } p=\frac{2}{k d-d+1}, \text { simplify } \\
& =r_{k, d},
\end{array}
$$

and so $G[U]$ also satisfies the weight requirement, and we are done.

Now, suppose that $G^{\prime} \cong K_{1,1,3}$ and $k=d=2$. Let $s$ be the number of partial vertices adjacent to $v$. Apply Remark 7 to obtain a 3 -vertex induced forest in $G^{\prime}$ that contains no vertices adjacent to $v$ in $G$.

If $s \geqslant 3$, then let $U$ consist of this 3-vertex set, together with the $s$ partial vertices. Since $k=d=2$, we have $p=2 / 3$. Substituting $w(U)=3+s p, w(G)=6+s p, p=2 / 3$, and $k=d=2$ into

$$
w(U) \geqslant \frac{2 d \cdot w(G)+2}{k d+d+1}
$$

and simplifying, we obtain $s \geqslant 5 / 2$, which holds when $s \geqslant 3$.

On the other hand, if $s=2$, then let $U$ consist of the 3-vertex set constructed above, plus $v$ and the 2 partial vertices. Then $w(U)=4+2 p$, while $w(G)=6+2 p$. Substituting and simplifying, as above, we can see that $w(U)$ is again large enough.

Henceforth, we assume that each full vertex of $G$ has fewer than $d$ partial neighbors.

Reduction B-Suppose that a vertex $v$ that is simplicial in $G-P$ has one or more partial neighbors in $G$. (See the second graph in Figure 1.)

Remove these partial neighbors, and let $G^{\prime}$ be the graph that remains. If $G^{\prime} \cong K_{1,1,3}$ and $k=d=2$, then let $U^{\prime}$ be a 3 -vertex independent set in $G^{\prime}$; otherwise, find $U^{\prime}$ by the induction hypothesis. Let $U$ be $U^{\prime}$ together with the removed partial vertices. Then

$$
\frac{w(U)-w\left(U^{\prime}\right)}{w(G)-w\left(G^{\prime}\right)}=1>r_{k, d}
$$

and so the strict weight inequality holds. 
We consider the degree requirement. If $v \in U^{\prime}$, then, since $v$ is simplicial in $G-P$, the degree of $v$ in the forest $G^{\prime}\left[U^{\prime}\right]$ is at most 1 . Therefore, since $v$ has fewer than $d$ partial neighbors in $G$, the degree of $v$ in $G[U]$ is at most $d$. Thus, the degree requirement is satisfied.

Henceforth, we assume that each vertex that is simplicial in $G-P$ has no partial neighbors in $G$.
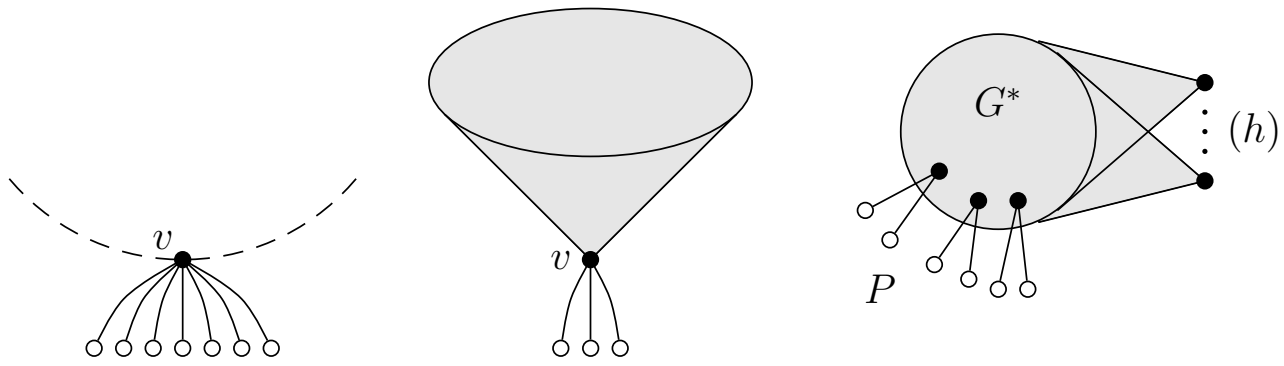

Figure 1: Reduction A, Reduction B, and Base Case 3. Partial vertices are shown as hollow vertices, and ovals represent cliques.

Base Case 2-Suppose that $G-P$ is a complete graph.

Since every vertex of $G-P$ is simplicial, by Reduction B there are no partial vertices. Thus, $G=G-P$ is a complete graph with from 2 to $k+1$ vertices. Let $U$ be any two vertices of $G$. Then $w(U)=2$, while $w(G) \leqslant k+1$. Thus,

$$
w(U)=\frac{2 d(k+1)+2}{d(k+1)+1} \geqslant \frac{2 d \cdot w(G)+2}{k d+d+1},
$$

and so the weight requirement is satisfied.

Henceforth we assume that $G-P$ is not a complete graph.

Let $G^{*}$ be the subgraph obtained from $G-P$ by removing every vertex that is simplicial in $G-P$. Because $G-P$ is connected, but not complete, it contains a vertex that is not simplicial, and so $V\left(G^{*}\right) \neq \varnothing$. Since $G^{*}$ is chordal, there must be a vertex that is simplicial in $G^{*}$.

Base Case 3-Suppose that every vertex that is simplicial in $G^{*}$ dominates $G-P$.

A simplicial vertex of $G^{*}$ dominates $G^{*}$, and so $G^{*}$ must be a complete graph. Then every vertex of $G^{*}$ is simplicial in $G^{*}$, and so each vertex of $G-P$ is adjacent to all of $V\left(G^{*}\right)$. Since $G-P$ is a $k$-tree but not a complete graph, its (two or more) simplicial vertices cannot be adjacent. Therefore, for each simplicial vertex of $G-P$, its $k$ neighbors in $G-P$ are precisely the set $V\left(G^{*}\right)$.

We conclude that $G-P$ is formed from $G^{*} \cong K_{k}$ and an independent set $V(G)-P$ $V\left(G^{*}\right)$ of at least 2 vertices, with every vertex of the former adjacent to every vertex of the latter. Each vertex in $G^{*}$ has at most $d-1$ partial neighbors in $G$ (by Reduction A), and each vertex in $V(G)-P-V\left(G^{*}\right)$ has no partial neighbors (by Reduction B). Let $h=\left|V(G)-P-V\left(G^{*}\right)\right|$, and let $t=|P|$. (See the last graph in Figure 1.) 
Subcase 3a. Suppose that some vertex $v$ of $G^{*}$ has at most $d-h$ partial neighbors in $G$.

Let $U$ consist of $v$, the $h$ independent-set vertices, and the $t$ partial vertices. Since $v$ has at most $d$ neighbors in $U$, the degree requirement is satisfied. Observe that $w(U)=$ $h+t p+1$ and $w(G)=h+t p+k$. Substituting these into

$$
w(U) \geqslant \frac{2 d \cdot w(G)+2}{k d+d+1}
$$

and simplifying, we obtain $h+t p \geqslant 1$, which holds, and so the weight requirement is satisfied as well.

Subcase 3b. Suppose that each vertex of $G^{*}$ has at least $d-h+1$ partial neighbors in $G$.

Let $U=V(G)-V\left(G^{*}\right)$. Observe that $w(U)=h+t p$ and $w(G)=h+t p+k$. Making these substitutions, and using $p=2 /(k d-d+1)$, we can show that the desired bound is equivalent to

$$
h+t p \geqslant 2+d p .
$$

Since $G^{*} \cong K_{k}$ and each vertex of $G^{*}$ has at least $d-h+1$ partial neighbors, we have $t \geqslant k(d-h+1)$. Thus, to verify the required bound it suffices to show that $h+k(d-h+1) p \geqslant 2+d p$. Substituting $p=2 /(k d-d+1)$, we can show that this is equivalent to

$$
(d-3) h(k-1)+h(k-2)+2(k-1) \geqslant 0 .
$$

This holds when $d \geqslant 3$, since $h, k \geqslant 2$.

Thus, for the remainder of Subcase $3 \mathrm{~b}$, we may assume that $d=2$. The inequality $h+t p \geqslant 2+d p$ becomes $h+t p \geqslant 2+2 p$. Since $h \geqslant 2$, this inequality holds if $t \geqslant 2$, and so we may assume that $t$ is 0 or 1 , and thus some vertex of $G^{*}$ has no partial neighbors in $G$. Since this subcase assumes that each vertex of $G^{*}$ has at least $d-h+1$ partial neighbors, we have $0 \geqslant d-h+1$, and so $h \geqslant d+1=3$.

We consider the possible values of $k$, when $d=2$. If $k \geqslant 3$, then $p \leqslant 2 / 5$, and so $h+t p \geqslant h \geqslant 3 \geqslant 2+2 p$, and we are done. On the other hand, if $k=2$, then $p=2 / 3$, and so $h+t p \geqslant 2+2 p$ if $t=1$ or if $h \geqslant 4$. The only remaining case for $d=2$ is that in which $k=2, h=3$, and $t=0$, in which case $G$ is $K_{1,1,3}$ and $P=\varnothing$. Then the maximum induced forest has weight 3 , which is less than our lower bound. However, this is not a problem, since, when $k=d=2$, the graph $K_{1,1,3}$ is an exception to our result.

Summary of Simple Cases-For the remainder of this proof, we assume that all of the following hold:

- $G$ is connected,

- $G-P$ is a $k$-tree,

- no vertex of $G$ is adjacent to more than $d-1$ partial vertices,

- each simplicial vertex of $G-P$ has no partial neighbors in $G$, and 
- there is a vertex that is simplicial in $G^{*}$ and that does not dominate $G-P$.

In the next portion of the proof, we will choose such a simplicial vertex $y$ and use it to construct what we call a "lobe of depth 2".

\subsection{Depth 2 Techniques}

Lobes and Depth - Suppose that $X \subseteq V(G-P)$ induces a $k$-clique. If removing $X$ disconnects $G-P$, then $X$ together with any component of $G-P-X$, is an $X$-lobe of $G-P$. If an induced subgraph is an $X$-lobe for some $X$, then it is a lobe; the clique induced by $X$ is its clique of attachment. Consider the following procedure, performed on a lobe $B$ of $G-P$ : remove all vertices of $B$ that are simplicial and that do not lie in $X$. The number of times we must perform this procedure, to leave only $X$ remaining, is the depth of $B$.

An $X$-lobe $B$ of $G$ is an $X$-lobe $B^{\prime}$ of $G-P$, together with all partial vertices adjacent to $V\left(B^{\prime}\right)-X$ with their incident edges. The depth of a lobe $B$ of $G$ is the depth of $B^{\prime}=B-P$ as a lobe of $G-P$.

The main portion of our proof consists of a number of cases in which we handle lobes of depth 2 .

A Lobe of Depth 2-Again, some vertex $y$ that is simplicial in $G^{*}$, does not dominate $G-P$. Let $X$ be the neighborhood of $y$ in $G^{*}$. Then $X$ induces a $k$-clique; this is the clique of attachment of a depth 2 lobe $B_{2}$ in $G$. Since $y$ dominates $B_{2}$, but not $G-P$, we see that $B_{2}-P$ is not all of $G-P$, as required.

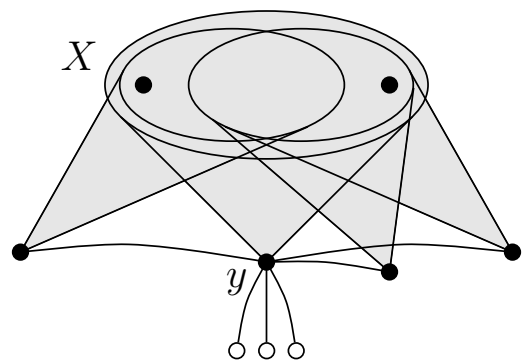

Figure 2: A lobe $B_{2}$ of depth 2, with $m=3, s=3$, and $q=2$. Note that $B_{2}-X$ is a star centered at $y$.

If we remove $X$ from the lobe $B_{2}$, then the resulting graph is a star centered at $y$ (partial neighbors of vertices in $X$ are not part of $B_{2}$.) Let $m$ be the number of full leaves of the star $B_{2}-X$. Then $m \geqslant 1$, since otherwise $y$ is simplicial in $G-P$. Each of these leaves is adjacent to $y$ and to exactly $k-1$ vertices in $X$. Let $s$ be the number of partial leaves of $B_{2}-X$, so that $0 \leqslant s \leqslant d-1$, by Reduction $\mathrm{A}$.

Label the elements of $X$ as $x_{1}, x_{2}, \ldots, x_{k}$. Let $q$ be the number of distinct sets of vertices in $B_{2}$ that are neighborhoods of full leaves of $B_{2}-X$. (For example, all full 
leaves of $B_{2}-X$ have the same neighborhood if and only if $q=1$.) Since $X$ has $k$ distinct subsets of size $k-1$, we have $q \leqslant k$.

See Figure 2 for an illustration of a depth 2 lobe.

We note that every depth 2 lobe of $G$ can be found in the above manner (by choosing a simplicial vertex in $G^{*}$ that does not dominate $G-P$, and so on).

Cases Involving Depth 2 Lobes-Having found a lobe $B_{2}$ of depth 2, we handle it based on $k$ and $d$, as well as the values of $m, s$, and $q$ (defined above) for $B_{2}$.

We split this portion of the proof into 14 cases that cover all possible values of $k, d$, $m, s$, and $q$, with $k, d \geqslant 2, m \geqslant 1,0 \leqslant s \leqslant d-1$, and $1 \leqslant q \leqslant \min \{k, m\}$.

(C1) $m=1$ and $s \leqslant d-2$.

(C2) $m=1$ and $s=d-1$.

(C3) $m=2$ and $s \geqslant 1$.

(C4) $k \geqslant 3, m \geqslant 2$, and $m+s \leqslant d$.

(C5) $k \geqslant 3, m \geqslant 3, m+s \geqslant d+1$, and either $m \geqslant 4$ or $k \geqslant 4$.

(C6) $k=3, m=3$, and $q \leqslant 2$.

(C7) $k=3, m=3$, and $q=3$.

(C8) $k=2, m=2, s=0$, and $d=2$.

(C9) $k=2, m=2, s=0, q=1$, and $d \geqslant 3$.

(C10) $k=2, m=2, s=0, q=2$, and $d \geqslant 3$.

(C11) $k=2, m \geqslant 3$, and $d \geqslant 3$.

(C12) $k=2, m=3, q=1$, and $d=2$.

(C13) $k=2, m=3, q=2$, and $d=2$.

(C14) $k=2, m \geqslant 4$, and $d=2$.

All the possibilities with $m=1$ are covered by $\mathrm{C} 1$ and $\mathrm{C} 2$. For $m=2$, if $s \geqslant 1$, then we are in Case C3; otherwise, for $k=2$ we are in one of $\mathrm{C} 8, \mathrm{C} 9, \mathrm{C} 10$, and for $k \geqslant 3$ we are in $\mathrm{C} 4$. For $k \geqslant 3$ and $m \geqslant 3$, Cases $\mathrm{C} 4$ and C5 cover all possibilities except for some with $k=m=3$, which are covered by Cases C6 and C7. For $k=2$ and $m \geqslant 3$, all possibilities are covered by Cases C11, C12, C13, and C14. (Some cases overlap.)

Thus, the 14 cases cover every possible value of $k, d, m, s$, and $q$.

Handling the Depth 2 Cases-We discuss how to handle each of the above cases, in the order listed.

We first give two facts, which can easily be verified.

Facts. For all $k \geqslant 2$ and $d \geqslant 2$, both of the following hold. 

(i) $\frac{2-p}{k+1-p}=r_{k, d}$.
(ii) $\frac{2+(d-1) p}{k+2+(d-1) p}=r_{k, d}$.

We now cover the cases in the order listed above.

Case C1. Suppose that $m=1$ and $s \leqslant d-2$.

Since $m=1$, there is one vertex in $X$ (say $x_{1}$ ) that is not adjacent to the full leaf of the star $B_{2}-X$. (See the first graph in Figure 3.) Remove all vertices of the lobe $B_{2}$, except for $x_{1}$ and $y$, and make $y$ partial, to obtain $G^{\prime}$. Find $U^{\prime}$ by the induction hypothesis, and let $U$ be $U^{\prime}$ plus all (full and partial) leaves of $B_{2}-X$. Note that $y \in U$. Then $G[U]$ is a forest satisfying the degree requirement. We check the weight requirement.

$$
\begin{aligned}
\frac{w(U)-w\left(U^{\prime}\right)}{w(G)-w\left(G^{\prime}\right)} & =\frac{s p+1+(1-p)}{s p+k+(1-p)} & & \\
& \geqslant \frac{2-p}{k+1-p} & & \text { minimized when } s=0 \\
& =r_{k, d} . & & \text { by Fact }(\mathrm{i})
\end{aligned}
$$

Since the construction of $G^{\prime}$ involved the creation of a new partial vertex, we do not need to verify the strict weight inequality. (We will generally not point this out in later cases.)

Case C2. Suppose that $m=1$ and $s=d-1$.

(See the first graph in Figure 3 for an illustration of this case.) Remove the entire lobe $B_{2}$ to obtain $G^{\prime}$. If $G^{\prime} ¥ K_{1,1,3}$ or $k>2$ or $d>2$, then find $U^{\prime}$ by the induction hypothesis, and let $U$ be $U^{\prime}$ plus all vertices in $B_{2}-X$. Then $G[U]$ is the disjoint union of $G^{\prime}\left[U^{\prime}\right]$ with a star of maximum degree $d$, and the weights satisfy

$$
\begin{aligned}
\frac{w(U)-w\left(U^{\prime}\right)}{w(G)-w\left(G^{\prime}\right)} & =\frac{2+s p}{k+2+s p} & \\
& =r_{k, d} . & \text { by Fact (ii), since } s=d-1
\end{aligned}
$$

If $G^{\prime} \cong K_{1,1,3}$ and $k=d=2$, then we apply Remark 7 to obtain a 3-vertex induced forest in $G^{\prime}$ that contains no vertices adjacent to $X$ in $G$. Let $U$ consist of these three vertices, $x_{1}, x_{2}$, the full leaf of the star $B_{2}-X$, and the partial vertex (there is only one, since $s=d-1=1)$. Then $w(U)=6+p$, while $w(G)=9+p$. Further, $k=d=2$, and so $p=2 / 3$. Substituting these into

$$
w(U) \geqslant \frac{2 d \cdot w(G)+2}{k d+d+1}
$$

and simplifying, we see that $U$ satisfies the weight requirement. (Note that here we constructed $U$ explicitly, $U^{\prime}$ was never defined, and there is no need to verify the strict weight inequality.) 

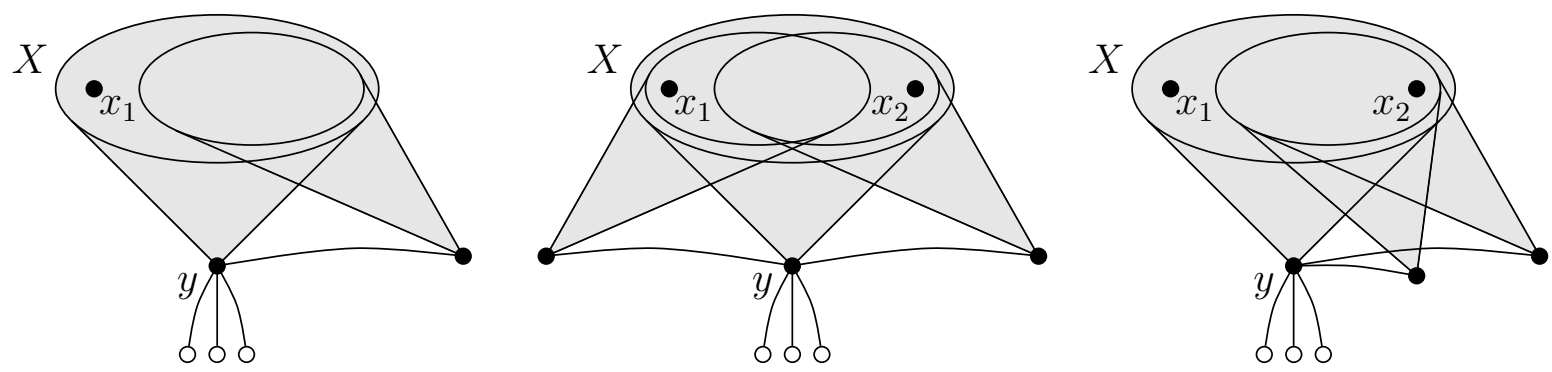

Figure 3: Case C1/C2, Case C3 with $q=2$, and Case C3 with $q=1$.

Case C3. Suppose that $m=2$ and $s \geqslant 1$.

Since $m=2$ we must have $q \leqslant 2$, and so there exist two distinct vertices of $X$ (say $\left.x_{1}, x_{2}\right)$, so that each full leaf of the star $B_{2}-X$ is adjacent to only one of these vertices. (See the second and third graphs in Figure 3.) Remove $y$, all partial leaves of $B_{2}-X$, and $X-\left\{x_{1}, x_{2}\right\}$, and make the full leaves partial, to obtain $G^{\prime}$. Find $U^{\prime}$ by the induction hypothesis, and let $U$ be $U^{\prime}$ plus all partial leaves of $B_{2}-X$. Note that both full leaves of $B_{2}-X$ also lie in $U$. We check the weight requirement.

$$
\begin{aligned}
\frac{w(U)-w\left(U^{\prime}\right)}{w(G)-w\left(G^{\prime}\right)} & =\frac{s p+2(1-p)}{s p+2(1-p)+1+(k-2)} & & \\
& \geqslant \frac{p+2(1-p)}{p+2(1-p)+1+(k-2)} & & \text { minimized when } s=1 \\
& =\frac{2-p}{k+1-p} & & \\
& =r_{k, d} . & & \text { by Fact (i) }
\end{aligned}
$$

Case C4. Suppose that $k \geqslant 3, m \geqslant 2$, and $m+s \leqslant d$.

Remove the entire lobe $B_{2}$ to obtain $G^{\prime}$. Find $U^{\prime}$ by the induction hypothesis, and let $U$ be $U^{\prime}$ plus all vertices in $B_{2}-X$. Then

$$
\begin{aligned}
\frac{w(U)-w\left(U^{\prime}\right)}{w(G)-w\left(G^{\prime}\right)} & =\frac{m+s p+1}{m+s p+k+1} & \\
& \geqslant \frac{3}{k+3} . & \text { minimized when } m=2 \& s=0
\end{aligned}
$$

The inequality

$$
\frac{3}{k+3} \geqslant \frac{2 d}{k d+d+1}
$$

simplifies to $k \geqslant 3-3 / d$, which holds, since $k \geqslant 3$.

Note that, while it is possible that $G^{\prime}$ contains no partial vertices, we always have $k \neq 2$ in Case $\mathrm{C} 4$, and so we do not need to treat $G^{\prime} \cong K_{1,1,3}$ separately. (We will generally not point this out in later cases.) 
Case C5. Suppose that $k \geqslant 3, m \geqslant 3, m+s \geqslant d+1$, and either $m \geqslant 4$ or $k \geqslant 4$.

Remove the entire lobe $B_{2}$ to obtain $G^{\prime}$. Find $U^{\prime}$ by the induction hypothesis, and let $U$ be $U^{\prime}$ plus all leaves of the star $B_{2}-X$. Then

$$
\frac{w(U)-w\left(U^{\prime}\right)}{w(G)-w\left(G^{\prime}\right)}=\frac{m+s p}{m+s p+k+1} .
$$

The inequality

$$
\frac{(m+s p)}{(m+s p)+k+1} \geqslant \frac{2 d}{k d+d+1}
$$

simplifies to

$$
m+s p \geqslant \frac{2(k+1) d}{k d-d+1} .
$$

We consider this inequality when $m \geqslant 4$, and then when $k \geqslant 4$.

Suppose that $m \geqslant 4$. It suffices to verify the inequality obtained by replacing $m+s p$ by 4 above. This simplifies to $k d \geqslant 3 d-2$, which holds, since $k \geqslant 3$.

Now suppose that $k \geqslant 4$. The quantity $m+s p$ is minimized when $m=3$ and $m+s=d+1$, that is, $s=d-2$. Thus, we replace $m+s p$ by $3+(d-2) p$ above, substitute $p=2 /(k d-d+1)$, and simplify to $(k-3) d \geqslant 1$, which holds, since $k \geqslant 4$ and $d \geqslant 2$.
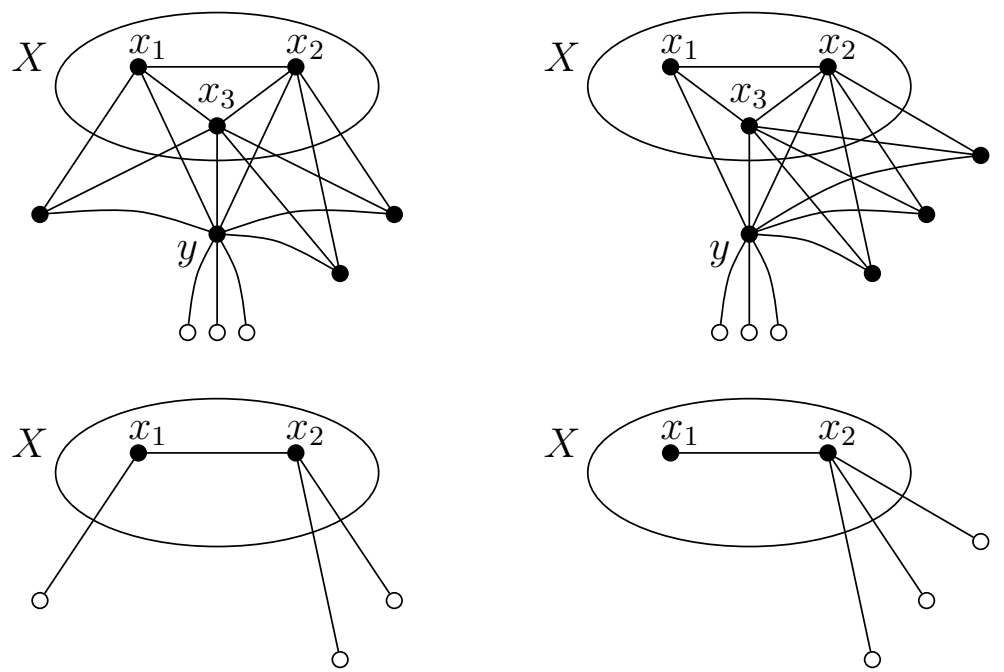

Figure 4: Case C6. On the left, $q=2$ (before and after). On the right, $q=1$ (before and after).

Case C6. Suppose that $k=3, m=3$, and $q \leqslant 2$.

Since $q \leqslant 2$, there is a vertex in $X$ (say $x_{3}$ ) that is adjacent to every full leaf of $B_{2}-X$. (See Figure 4.) Then each full leaf is adjacent to exactly one vertex in $\left\{x_{1}, x_{2}\right\}$. Remove $x_{3}, y$, and all partial leaves, and make all the full leaves partial, to obtain $G^{\prime}$. Find $U^{\prime}$ by the induction hypothesis, and let $U$ be $U^{\prime}$ plus all partial leaves. Note that all full leaves 
also lie in $U$. Thus,

$$
\begin{aligned}
\frac{w(U)-w\left(U^{\prime}\right)}{w(G)-w\left(G^{\prime}\right)} & =\frac{3(1-p)+s p}{3(1-p)+s p+2} & \\
& \geqslant \frac{3-3 p}{5-3 p} . & \text { minimized when } s=0
\end{aligned}
$$

Thus, it suffices to show that

$$
\frac{3-3 p}{5-3 p} \geqslant \frac{2 d}{k d+d+1}
$$

Substituting $p=2 /(k d-d+1)$ and $k=3$, this inequality simplifies to $(2 d-3)(2 d+1) \geqslant 0$, which holds, since $d \geqslant 2$.

Case C7. Suppose that $k=3, m=3$, and $q=3$.
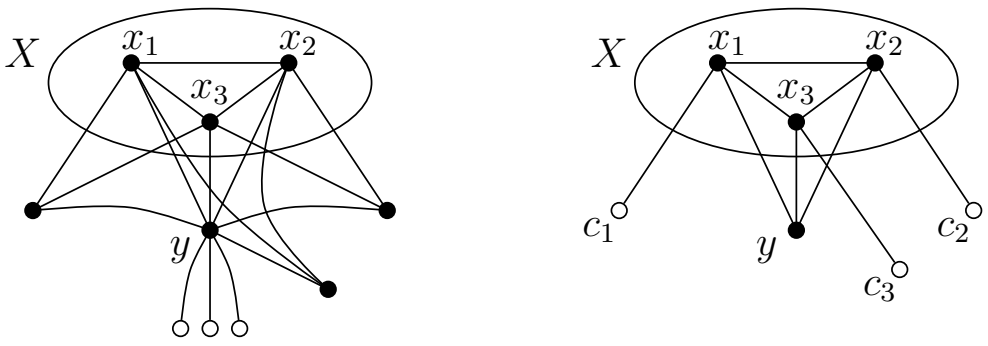

Figure 5: Case C7 (before and after).

Remove all full and partial leaves of the star $B_{2}-X$, and add new partial vertices $c_{1}$, $c_{2}, c_{3}$, so that $c_{i}$ is adjacent only to $x_{i}$, for $i=1,2,3$. Let $G^{\prime}$ be the resulting graph. (See Figure 5.) Find $U^{\prime}$ by the induction hypothesis.

We may assume that $y \in U^{\prime}$. If it is not, then we can add $y$ to $U^{\prime}$, removing one element of $X$ from $U^{\prime}$ if $U^{\prime}$ meets $X$, to obtain a new $U^{\prime}$ that contains $y$. Note that now $U^{\prime}$ contains at most 1 vertex of $X$.

Let $U$ be $U^{\prime}-\left\{c_{1}, c_{2}, c_{3}, y\right\}$ plus all leaves of $B_{2}-X$. If $x_{i} \in U^{\prime}$ then its degree is the same in $G[U]$ as in $G\left[U^{\prime}\right]$; hence, $G[U]$ is a forest of maximum degree at most $d$.

For the weight requirement, we have

$$
\begin{aligned}
\frac{w(U)-w\left(U^{\prime}\right)}{w(G)-w\left(G^{\prime}\right)} & =\frac{3+s p-3 p-1}{3+s p-3 p} & \\
& \geqslant \frac{2-3 p}{3-3 p} . & \text { minimized when } s=0
\end{aligned}
$$

Thus, it suffices to show that

$$
\frac{2-3 p}{3-3 p} \geqslant \frac{2 d}{k d+d+1}
$$


Substituting $p=2 /(k d-d+1)$ and $k=3$, this inequality simplifies to $(2 d+1)(d-2) \geqslant 0$, which holds, since $d \geqslant 2$.

Case C8. Suppose that $k=2, m=2, s=0$, and $d=2$.

Remove the entire lobe $B_{2}$ to obtain $G^{\prime}$. If $G^{\prime} \cong K_{1,1,3}$, then let $U^{\prime}$ be a 3 -vertex independent set in $G^{\prime}$; otherwise, find $U^{\prime}$ by the induction hypothesis. Let $U$ be $U^{\prime}$ plus all vertices in $B_{2}-X$. Then

$$
\begin{aligned}
\frac{w(U)-w\left(U^{\prime}\right)}{w(G)-w\left(G^{\prime}\right)} & =\frac{3}{5} \\
& >\frac{4}{7}=r_{k, d}, \quad k=2 \& d=2
\end{aligned}
$$

and so the strict weight inequality holds.

Case C9. Suppose that $k=2, m=2, s=0, q=1$, and $d \geqslant 3$.

Since $q=1$, there is one vertex in $X$ (say $x_{1}$ ) that is not adjacent to either leaf of the star $B_{2}-X$. (See the first graph in Figure 6.) Remove $x_{2}$ and the two leaves of $B_{2}-X$, and make $y$ partial, to obtain $G^{\prime}$. Find $U^{\prime}$ by the induction hypothesis, and let $U$ be $U^{\prime}$ plus all leaves of $B_{2}-X$. Note that $y \in U$. Since $d \geqslant 3$, the degree requirement is satisfied. We have

$$
\begin{array}{rlr}
\frac{w(U)-w\left(U^{\prime}\right)}{w(G)-w\left(G^{\prime}\right)} & =\frac{2+(1-p)}{3+(1-p)} & \\
& >\frac{2-p}{3-p} & \\
& =r_{k, d} . & \text { by Fact (i), since } k=2
\end{array}
$$

Case C10. Suppose that $k=2, m=2, s=0, q=2$, and $d \geqslant 3$. (See Figure 7.)

To deal with this case we will use techniques involving lobes of depth 3 . We discuss these later, in Subsection 2.3.

Case C11. Suppose that $k=2, m \geqslant 3$, and $d \geqslant 3$.

Remove $y$ and all partial leaves of the star $B_{2}-X$, and make all the full leaves partial, to obtain $G^{\prime}$. Find $U^{\prime}$ by the induction hypothesis, and let $U$ be $U^{\prime}$ plus all partial leaves of $B_{2}-X$. Note that all full leaves of $B_{2}-X$ also lie in $U$. Thus,

$$
\begin{aligned}
\frac{w(U)-w\left(U^{\prime}\right)}{w(G)-w\left(G^{\prime}\right)} & =\frac{s p+m(1-p)}{s p+m(1-p)+1} & \\
& \geqslant \frac{3-3 p}{4-3 p} . & \text { minimized when } m=3 \& s=0
\end{aligned}
$$

Thus, it suffices to show that

$$
\frac{3-3 p}{4-3 p} \geqslant \frac{2 d}{k d+d+1}
$$


Substituting $p=2 /(k d-d+1)$ and $k=2$, this inequality simplifies to $(d-3)(d+1) \geqslant 0$, which holds, since $d \geqslant 3$.

Case C12. Suppose that $k=2, m=3, q=1$, and $d=2$.
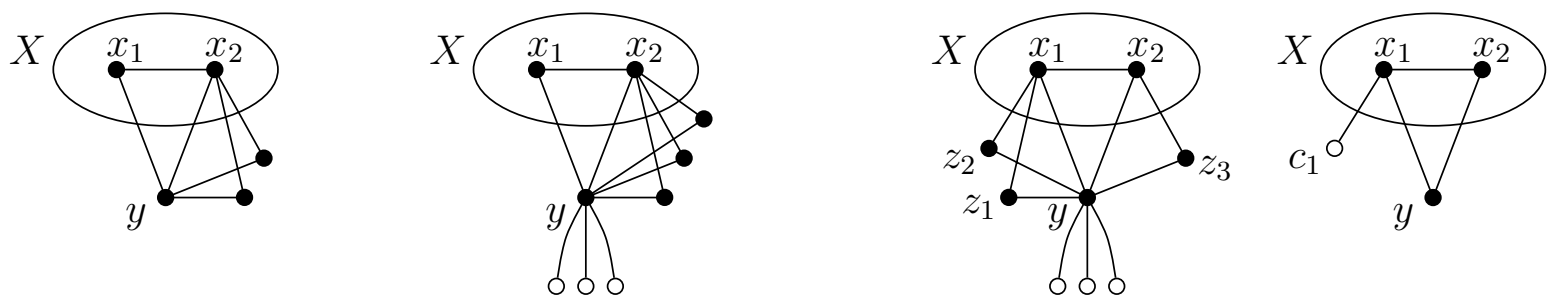

Figure 6: Case C9, Case C12, and Case C13 (before and after).

Since $q=1$, there is one vertex in $X$ (say $x_{1}$ ) that is not adjacent to any full leaf of the star $B_{2}-X$. (See the second graph in Figure 6.) Remove all vertices of $B_{2}$, except for $x_{1}$, to obtain $G^{\prime}$. If $G^{\prime} \cong K_{1,1,3}$, then let $U^{\prime}$ be a 3 -vertex independent set in $G^{\prime}$; otherwise, find $U^{\prime}$ by the induction hypothesis. Let $U$ be $U^{\prime}$ plus all leaves of $B_{2}-X$. Then

$$
\begin{aligned}
\frac{w(U)-w\left(U^{\prime}\right)}{w(G)-w\left(G^{\prime}\right)} & =\frac{3+s p}{5+s p} & & \\
& \geqslant \frac{3}{5} & & \text { minimized when } s=0 \\
& >\frac{4}{7}=r_{k, d}, & & k=2 \& d=2
\end{aligned}
$$

and so the strict weight inequality holds.

Case C13. Suppose that $k=2, m=3, q=2$, and $d=2$.

Let $z_{1}, z_{2}, z_{3}$ be the full leaves of the star $B_{2}-X$. Without loss of generality, we may assume that $N\left(z_{1}\right)=N\left(z_{2}\right)=\left\{x_{1}, y\right\}$, while $N\left(z_{3}\right)=\left\{x_{2}, y\right\}$. Remove all full and partial leaves of $B_{2}-X$, and add a partial vertex $c_{1}$ adjacent to $x_{1}$. Let $G^{\prime}$ be the resulting graph. (See the last two graphs in Figure 6.) Find $U^{\prime}$ by the induction hypothesis.

We may assume that $y \in U^{\prime}$. If it is not, then we can add $y$ to $U^{\prime}$, removing one element of $X$ from $U^{\prime}$ if $U^{\prime}$ meets $X$, to obtain a new $U^{\prime}$ that contains $y$. Note that now $U^{\prime}$ contains at most 1 vertex of $X$.

Let $U$ be $U^{\prime}-\left\{c_{1}, y\right\}$ plus all leaves of the star $B_{2}-X$. If $x_{i} \in U^{\prime}$ then its degree is the same in $G[U]$ as in $G\left[U^{\prime}\right]$; hence, $G[U]$ is a forest of maximum degree at most $d$. Then

$$
\begin{aligned}
\frac{w(U)-w\left(U^{\prime}\right)}{w(G)-w\left(G^{\prime}\right)} & =\frac{3+s p-p-1}{3+s p-p} & & \\
& \geqslant \frac{2-p}{3-p} & & \text { minimized when } s=0 \\
& =r_{k, d} . & & \text { by Fact (i), since } k=2
\end{aligned}
$$

Case C14. Suppose that $k=2, m \geqslant 4$, and $d=2$. 
Remove the entire lobe $B_{2}$, to obtain $G^{\prime}$. First, suppose that $G^{\prime} ¥ K_{1,1,3}$. In this case, find $U^{\prime}$ by the induction hypothesis, and let $U$ be $U^{\prime}$ plus all leaves of $B_{2}-X$. Then

$$
\begin{aligned}
\frac{w(U)-w\left(U^{\prime}\right)}{w(G)-w\left(G^{\prime}\right)} & =\frac{m+s p}{m+s p+3} & \\
& \geqslant \frac{m}{m+3} . & \text { minimized when } s=0
\end{aligned}
$$

Since $m \geqslant 4$, this fraction is at least $4 / 7=r_{k, d}$.

Now suppose that $G^{\prime} \cong K_{1,1,3}$. Applying Remark 7 , we let $U^{\prime}$ be a 3 -vertex set inducing a forest in $G^{\prime}$ that contains no vertices adjacent to $X$ in $G$.

If $m \geqslant 5$, then let $U$ be $U^{\prime}$ plus all leaves of $B_{2}-X$. Then $w(U)=3+m+s p$, while $w(G)=8+m+s p$. Substituting these, along with $k=d=2$, into

$$
w(U) \geqslant \frac{2 d \cdot w(G)+2}{k d+d+1}
$$

and simplifying, we obtain $m+s p \geqslant 13 / 3$, which holds, since $m \geqslant 5$.

Now suppose that $m=4$. Since $k=2$ and $m=4$, some element of $X$ (say $x_{1}$ ) is adjacent to at most 2 full leaves of $B_{2}-X$. Let $U$ be $U^{\prime}$ plus $x_{1}$ and all leaves of $B_{2}-X$. Then $w(U)=8+s p$, while $w(G)=12+s p$. Substituting and simplifying, as above, we obtain $s p \geqslant-2$, which holds.

Thus, $U$ satisfies the weight requirement.

Cases Covered by the Depth 2 Techniques-When $k \geqslant 3$ or $d=2$, we have handled every lobe of depth 2 , and so our proof of these cases is complete.

When $k=2$ and $d \geqslant 3$, we have handled every lobe of depth 2 except for that with $m=2, s=0$, and $q=2$ (Case C10; see Figure 7). Next we consider graphs in which every depth 2 lobe is of this type.

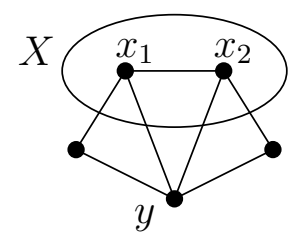

Figure 7: A depth 2 lobe of type C10.

\subsection{Depth 3 Techniques}

We have handled all depth 2 cases except for C10. Henceforth, we assume that every lobe of depth 2 in $G$ is in Case C10, that is, that $k=2, d \geqslant 3$, and each depth 2 lobe contains no partial vertices $(s=0)$ and two simplicial vertices $(m=2)$ having distinct neighborhoods $(q=2)$. (See Figure 7.)

Base Case 4-Suppose that $G$ has no lobe of depth 3 or more. 
We claim that $G$ must be isomorphic to the graph with vertex set $\left\{a_{1}, a_{2}, a_{3}, b_{1}, b_{2}, b_{3}\right\}$, in which the $a_{i}$ vertices form a triangle, the $b_{i}$ vertices form an independent set, and $a_{i}$ is adjacent to $b_{j}$ if and only if $i \neq j$. (This graph is known as the Hajós graph; it is pictured in Figure 8.)

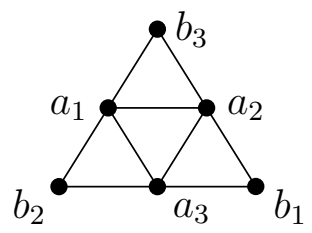

Figure 8: Base Case 4, the Hajós graph.

To see this, note that $G$ has a depth 2 lobe $B$, which must be in Case C10. Let $X=\left\{x_{1}, x_{2}\right\}$ be the clique of attachment of $B$, and let $y$ be the common neighbor of $x_{1}$, $x_{2}$ in $B$. Since $B$ is not all of $G-P$, there is another $X$-lobe $B^{\prime}$. If $B^{\prime}$ has depth 2 or more, then there is an $\left\{x_{1}, y\right\}$-lobe of depth 3 or more, contradicting our assumption. Thus, $B^{\prime}$ has depth 1 . If there is a third $X$-lobe, or if $x_{2}$ has any partial neighbors, then we have an $\left\{x_{1}, y\right\}$-lobe of depth 2 that is not in Case C10, another contradiction. Similarly, $x_{1}$ has no partial neighbors. We conclude that $G$ is the union of $B$ (depth 2, Case C10) and $B^{\prime}$ (depth 1), and that $P=\varnothing$. Hence, $G$ is the Hajós graph, with $\left\{a_{1}, a_{2}, a_{3}\right\}=\left\{x_{1}, x_{2}, y\right\}$.

To deal with this case, let $U=V(G)-\left\{a_{1}, a_{2}\right\}$. Then $w(U)=4$, while $w(G)=6$. Thus,

$$
w(U)=\frac{12 d+4}{3 d+1}>\frac{12 d+2}{3 d+1}=\frac{2 d \cdot w(G)+2}{k d+d+1},
$$

and so the weight requirement is satisfied.

Henceforth, we assume that $G$ has a lobe of depth 3 or more.

A Lobe of Depth 3-A lobe of depth more than 3 must contain a lobe of depth 3 . Thus, $G$ has a lobe $B_{3}$ of depth 3 . Let $X=\left\{x_{1}, x_{2}\right\}$ be the clique of attachment of $B_{3}$. Let $y$ be the common neighbor in $B_{3}$ of $x_{1}, x_{2}$. Let $t$ be the number of partial vertices adjacent to $y$; there can be no other partial vertices in $B_{3}$.

Reduction D-Suppose that $B_{3}$ contains two or more lobes of depth at most 2, at least one of which has depth exactly 2 , having the same clique of attachment $X^{\prime}$.

Remove the above $X^{\prime}$-lobes, to obtain $G^{\prime}$. This $G^{\prime}$ will have at least 1 full vertex, since the two $X^{\prime}$-lobes are contained in $B_{3}$ and, being a lobe, $B_{3}$ cannot contain $G-P$. Thus, we can find $U^{\prime}$ by the induction hypothesis, and let $U$ be $U^{\prime}$ plus all vertices of the removed lobes, except for those in $X^{\prime}$. Then the degree requirement is satisfied. Further, the fraction

$$
\frac{w(U)-w\left(U^{\prime}\right)}{w(G)-w\left(G^{\prime}\right)}
$$

is $6 / 8$ if both removed lobes have depth 2 , and $4 / 6$ otherwise; in either case, it is at least $r_{k, d}$ (since, when $k=2$, we have $\left.r_{k, d}=2 d /(3 d+1)<2 / 3\right)$, and so the weight requirement is also satisfied. 
Henceforth, we assume that, given a depth 2 lobe contained in $B_{3}$, there is no other lobe of depth at most 2 having the same clique of attachment.

Cases Involving Depth 3 Lobes - Having found a lobe $B_{3}$ of depth 3, we handle it based on the depth 1 and depth 2 lobes that it contains.

By Reduction D, no two depth 2 lobes in $B_{3}$ can have the same clique of attachment. Thus, $B_{3}$ must contain either 1 or 2 lobes of depth 2 ; if the latter, then one is attached at $\left\{x_{1}, y\right\}$, and the other is attached at $\left\{x_{2}, y\right\}$. Further, if a depth 2 lobe is attached at a 2-clique, then no depth 1 lobe is attached at this same clique.

We split this portion of the proof into 3 cases, listed below. We observe that these cases cover all the possibilities.

(E1) $B_{3}$ contains two lobes of depth 2 .

(E2) $B_{3}$ contains exactly one lobe of depth 2 , and no full simplicial vertices other than those in the lobe of depth 2.

(E3) $B_{3}$ contains exactly one lobe of depth 2 , and at least one full simplicial vertex other than those in the lobe of depth 2 .

Handling the Depth 3 Cases-We discuss how to handle each of the above cases, in the order listed.

Case E1. Suppose that $B_{3}$ contains two lobes of depth 2.

Then $B_{3}$ has exactly 9 full vertices. By Reduction $\mathrm{D}$, one of the depth 2 lobes must be attached at $\left\{x_{1}, y\right\}$, and the other at $\left\{x_{2}, y\right\}$, (See the first graph in Figure 9.)

Delete $B_{3}$ to obtain $G^{\prime}$. Find $U^{\prime}$ by the induction hypothesis, and let $U$ be $U^{\prime}$ plus all vertices of $B_{3}$ except for $x_{1}, x_{2}$, and $y$. Recall that $t$ is the number of partial vertices adjacent to $y$. Thus,

$$
\frac{w(U)-w\left(U^{\prime}\right)}{w(G)-w\left(G^{\prime}\right)}=\frac{6+t p}{9+t p} \geqslant \frac{6}{9}>r_{k, d},
$$

and so the weight requirement is satisfied.

In the remaining cases (E2 and E3), $B_{3}$ contains exactly one lobe of depth 2 . We may assume, without loss of generality, that its clique of attachment is $\left\{x_{1}, y\right\}$. Label its full simplicials as $z_{1}, z_{2}$, and its remaining vertex as $y^{\prime}$, so that $N\left(z_{1}\right)=\left\{x_{1}, y^{\prime}\right\}$, $N\left(z_{2}\right)=\left\{y^{\prime}, y\right\}$, and $N\left(y^{\prime}\right)=\left\{x_{1}, y, z_{1}, z_{2}\right\}$. (See the second graph in Figure 9 and the first graph in Figure 10.)

Case E2. Suppose that $B_{3}$ contains exactly one lobe of depth 2, and no full simplicial vertices other than those in the lobe of depth 2 .

Then $B_{3}$ has exactly 6 full vertices. (See the second graph in Figure 9.) By Reduction $\mathrm{A}, t \leqslant d-1$. We have two subcases: Subcase E2a, in which $t \leqslant d-2$, and Subcase E2b, in which $t=d-1$.

Subcase E2a. Suppose that $t \leqslant d-2$. 

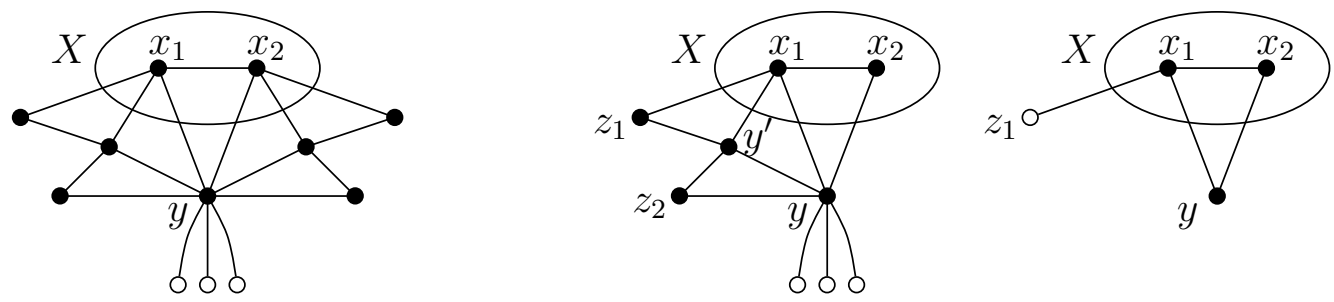

Figure 9: Case E1 and Case E2 (before and, for Subcase E2a, after).

Delete the $t$ partial vertices, $z_{2}$, and $y^{\prime}$, and make $z_{1}$ partial. Let $G^{\prime}$ be the resulting graph. (See the last graph in Figure 9.) Find $U^{\prime}$ by the induction hypothesis, and let $U$ be $U^{\prime}$ plus the $t$ partial vertices and $z_{2}$. Note that $z_{1} \in U$. If $y \in U^{\prime}$, then the degree of $y$ in $G^{\prime}\left[U^{\prime}\right]$ is at most one, and so the degree of $y$ in $G[U]$ is at most $t+2 \leqslant d$. It follows that $G[U]$ is a forest that satisfies the degree requirement; we verify the weight requirement.

$$
\begin{aligned}
\frac{w(U)-w\left(U^{\prime}\right)}{w(G)-w\left(G^{\prime}\right)} & =\frac{1+t p+(1-p)}{2+t p+(1-p)} \\
& \geqslant \frac{2-p}{3-p} \\
& =r_{k, d} .
\end{aligned}
$$

minimized when $t=0$

by Fact (i), since $k=2$

Subcase E2b. Suppose that $t=d-1$.

Delete every vertex of $B_{3}$, except for $x_{2}$, to obtain $G^{\prime}$. Find $U^{\prime}$ by the induction hypothesis, and let $U$ be $U^{\prime}$ plus $y^{\prime}, z_{1}, z_{2}$, and the $d-1$ partial vertices. Then

$$
\begin{aligned}
\frac{w(U)-w\left(U^{\prime}\right)}{w(G)-w\left(G^{\prime}\right)} & =\frac{3+(d-1) p}{5+(d-1) p} \\
& >\frac{2+(d-1) p}{4+(d-1) p} \\
& =r_{k, d} .
\end{aligned}
$$

by Fact (ii), since $k=2$
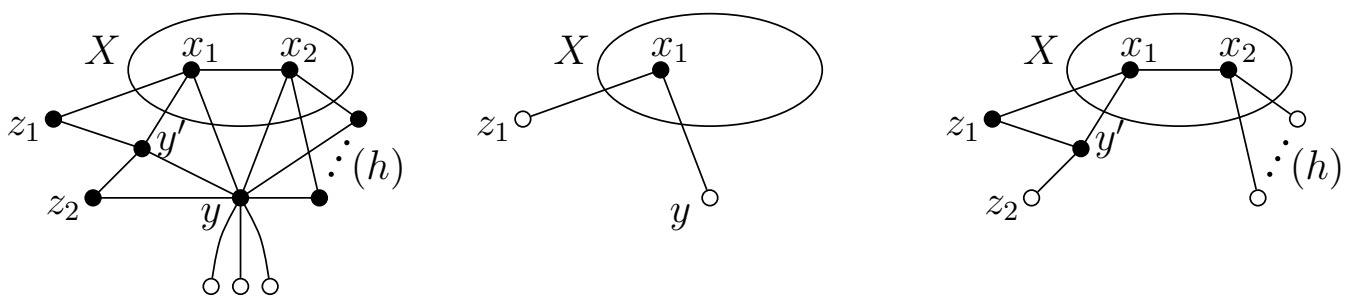

Figure 10: Case E3 (left) and results of Subcases E3a (center) and E3b (right).

Case E3. Suppose that $B_{3}$ contains exactly one lobe of depth 2, and at least one full simplicial vertex other than those in the lobe of depth 2 . 
Let $h$ be the number of full simplicials outside of the depth 2 lobe; each of these has neighborhood $\left\{x_{2}, y\right\}$. (See the first graph in Figure 10.) Then $B_{3}$ has exactly $h+6$ full vertices. We have two subcases: Subcase E3a, in which $h+t \leqslant d-2$, and Subcase E3b, in which $h+t \geqslant d-1$.

Subcase E3a. Suppose that $h+t \leqslant d-2$.

Delete the $t$ partial vertices, $x_{2}, y^{\prime}, z_{2}$, and the $h$ full simplicials, and make $y$ and $z_{1}$ partial. Let $G^{\prime}$ be the resulting graph. (See the second graph in Figure 10.) Find $U^{\prime}$ by the induction hypothesis, and let $U$ be $U^{\prime}$ plus $z_{2}$, the $h$ full simplicials, and the $t$ partial vertices. Note that $y, z_{1} \in U$. Thus,

$$
\begin{aligned}
\frac{w(U)-w\left(U^{\prime}\right)}{w(G)-w\left(G^{\prime}\right)} & =\frac{h+1+t p+2(1-p)}{h+3+t p+2(1-p)} & & \\
& \geqslant \frac{2+2(1-p)}{4+2(1-p)} & & \text { minimized when } h=1 \& t=0 \\
& =\frac{2-p}{3-p} & & \\
& =r_{k, d} . & & \text { by Fact }(\mathrm{i}), \text { since } k=2
\end{aligned}
$$

Subcase E3b. Suppose that $h+t \geqslant d-1$.

Delete $y$ and the $t$ partial vertices, and make the $h$ full simplicials and $z_{2}$ partial. Let $G^{\prime}$ be the resulting graph. (See the last graph in Figure 10.) Find $U^{\prime}$ by the induction hypothesis, and let $U$ be $U^{\prime}$ plus the $t$ partial vertices. Note that $U$ contains the $h$ full simplicials and $z_{2}$. Then

$$
\frac{w(U)-w\left(U^{\prime}\right)}{w(G)-w\left(G^{\prime}\right)}=\frac{t p+(h+1)(1-p)}{t p+(h+1)(1-p)+1} .
$$

We minimize the right-hand size above by setting $t=d-2$ and $h=1$. To see this, note that, when $d \geqslant 3$, we have $1-p \geqslant p$. Thus, to minimize $t p+(h+1)(1-p)$, while maintaining $h+t \geqslant d-1$, we set $h$ as small as possible $(h=1)$ and $t$ only as large as necessary $(t=d-1-h=d-2)$. Continuing:

$$
\begin{aligned}
& \geqslant \frac{(d-2) p+2(1-p)}{(d-2) p+2(1-p)+1} \\
& \geqslant \frac{p+2(1-p)}{p+2(1-p)+1} \quad \text { minimized when } d=3 \\
& =\frac{2-p}{3-p} \\
& =r_{k, d} \text {. }
\end{aligned}
$$

by Fact (i), since $k=2$

This completes the proof of Lemma 6.

We have now proven Theorem 1. In the next section we consider sharpness of this result. 


\section{Sharpness of Theorem 1}

We construct examples to show that, for each $k, d \geqslant 2$, the bound of Theorem 1 is tight to within 1 for all $n \geqslant 1$, and exactly sharp for infinitely many values of $n$.

Example 8. We construct a graph $G(k, d, n)$ for each $n \geqslant 1$ and $k, d \geqslant 2$.

If $n \leqslant k+1$, then let $G(k, d, n)=K_{n}$.

For the case when $n \geqslant k+2$, choose nonnegative integers $a, h$ with $h \leqslant k d+d$. Let $n=k+2+a(k d+d+1)+h$. Note that each $n \geqslant k+2$ is obtained from a unique pair $a, h$.

Let $b=\lfloor h /(k+1)\rfloor$, and let $c=h-b(k+1)$, so that $h=b(k+1)+c, 0 \leqslant b \leqslant d$, and $0 \leqslant c \leqslant k$.

Now we construct $G(k, d, n)$. Let $z_{0}, \ldots, z_{a}$ be distinct vertices. For each pair $(i, j)$ with $1 \leqslant i \leqslant a$ and $1 \leqslant j \leqslant d$, for each pair $(i, j)$ with $i=a+1$ and $1 \leqslant j \leqslant b$, and for $(i, j)=(1,0)$, let $S_{i, j}$ be a $k$-clique, with every vertex adjacent to $z_{i-1}$ and to another vertex $x_{i, j}$. If $c \neq 0$, then let $S^{\prime}$ be a $c$-clique, each of whose vertices is adjacent to $z_{a}$. Note that we have specified $(a+1)+(a d+b+1)(k+1)+c=n$ vertices. In each $S_{i, j}$, choose a vertex $y_{i, j}$. For each $1 \leqslant i \leqslant a$, let $z_{i}$ be adjacent to every vertex in $S_{i, d}-y_{i, d}+x_{i, d}$.

This defines $G(k, d, n)$. See Figures 11 and 12 for illustrations.

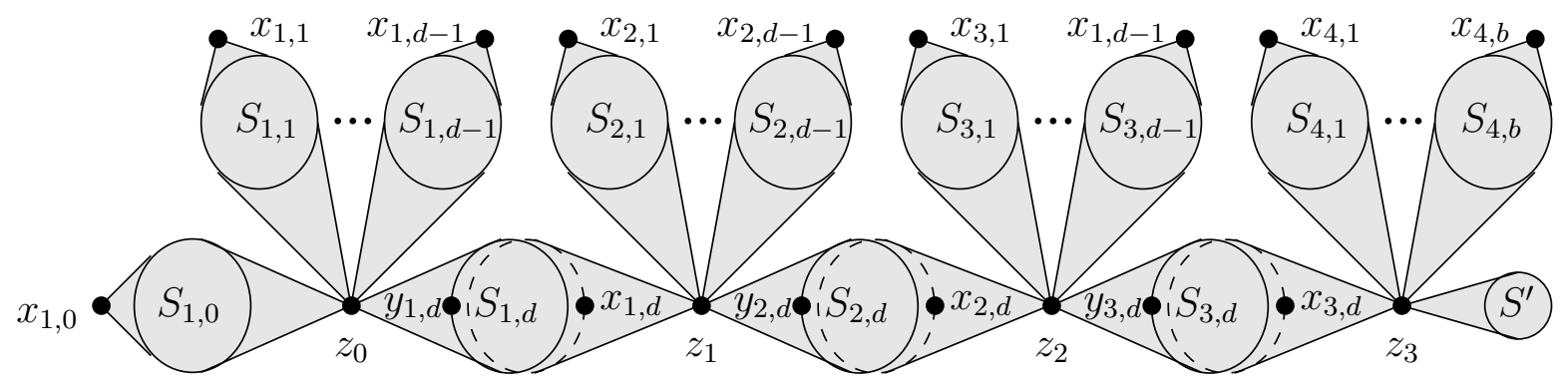

Figure 11: The structure of $G(k, d, n)$ with $a=3$. Circles represent $k$-cliques. Although every $k$-clique $S_{i, j}$ contains a vertex $y_{i, j}$, those vertices are only indicated in the figure when both $i \leqslant a$ and $j=d$.

Now we investigate $f_{d}(G(k, d, n))$.

Proposition 9. Let $k, d \geqslant 2$. Then the following both hold.

(i) For each $n \geqslant 1$, the graph $G(k, d, n)$, from Example 8, has order $n$ and treewidth at most $k$, and

$$
f_{d}(G(k, d, n)) \leqslant 1+\left\lceil\frac{2 d n+2}{k d+d+1}\right\rceil=1+\left\lceil r_{k, d} \cdot n+\frac{r_{k, d}}{d}\right\rceil .
$$




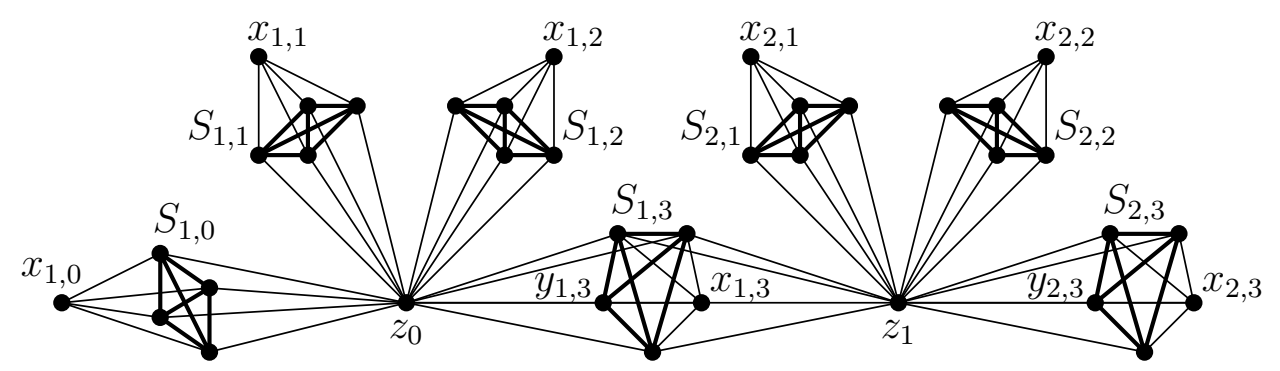

Figure 12: $G(k, d, n)$ with $n=37, k=4, d=3$; thus $a=1, h=k d+d=15, b=d=3$, and $c=0$. Edges within each $S_{i, j}$ are shown in bold.

(ii) There exist infinitely many values of $n \geqslant 1$ for which

$$
f_{d}(G(k, d, n))=\left\lceil\frac{2 d n+2}{k d+d+1}\right\rceil=\left\lceil r_{k, d} \cdot n+\frac{r_{k, d}}{d}\right\rceil .
$$

Proof. Let $G=G(k, d, n)$. It follows immediately from the construction that the order of $G$ is $n$. Since this graph is chordal, its treewidth is 1 less than its clique number. If $n \leqslant k+1$, then the graph is complete, and its treewidth is $n-1 \leqslant k$. If $n \geqslant k+2$, then each $S_{i, j}+x_{i, j}$ induces a maximum clique of order $k+1$, and the treewidth is $k$.

Suppose that $n \leqslant k+1$. The expression $\lceil(2 d n+2) /(k d+d+1)\rceil$ has positive numerator and denominator, and so its value is at least 1 . Since $G \cong K_{n}$, an induced forest can have at most two vertices, and so statement (i) of the proposition holds for these values of $n$.

Now suppose that $n \geqslant k+2$. Let $a, h, b$, and $c$ be as in Example 8. We will refer to an induced forest of maximum degree at most $d$ and maximum order, as an optimal forest.

Note that if there are vertices $v, v^{\prime}$ in $G$ with $N(v)-v^{\prime} \subseteq N\left(v^{\prime}\right)-v$, and if there is an optimal forest that contains $v^{\prime}$ but not $v$, then, by removing $v^{\prime}$ and adding $v$, we obtain another optimal forest that contains $v$ but not $v^{\prime}$. Thus, if $v_{1}, v_{2}, v_{3}$ are vertices of a triangle for which $N\left(v_{1}\right)-v_{3} \subseteq N\left(v_{3}\right)-v_{1}$ and $N\left(v_{2}\right)-v_{3} \subseteq N\left(v_{3}\right)-v_{2}$, then we can find an optimal forest that does not contain $v_{3}$.

Applying these ideas to triples in each $S_{i, j}+x_{i, j}$, it follows that there is an optimal forest $T$ that does not meet any $S_{i, j}-y_{i, j}$. If $\left|S^{\prime}\right| \geqslant 2$, then by applying the ideas to triples in $S^{\prime}+z_{a}$, we may assume that $T$ does not contain $z_{a}$ and $T$ contains at most 2 vertices of $S^{\prime}$.

Removing from consideration those vertices that have been excluded from $T$, we are left with a set containing $z_{i}$ for $i<a$, all $x_{i, j}$ and $y_{i, j}$, and $\min \left\{2,\left|S^{\prime}+z_{1}\right|\right\}$ vertices of $S^{\prime}+z_{a}$. The number of $x_{i, j}$ vertices is $a d+b+1$; the number of $y_{i, j}$ vertices is the same. Let $W$ be this set of vertices, so that $V(T) \subseteq W$. Then

$$
|W| \leqslant a+2[a d+b+1]+2=a+2 a d+2 b+4 .
$$

Each $z_{i}$ with $i<a$ has degree $d+1$ in $G[W]$. Further, in $G[W]$, the sets $N\left(z_{i}\right)+z_{i}$ for $0 \leqslant i<a$, are pairwise disjoint; $T$ thus misses at least one vertex from each of these $a$ sets. Subtracting $a$, we obtain an upper bound of $2 a d+2 b+4$ for the order of $T$. 
If $c=0$, then we can do better, since $S^{\prime}$ has no vertices, and so $T$ has order at most $2 a d+2 b+3$. If $h=k d+d$, then $c=0$ and $b=d$, and we can do better still. Because $S^{\prime}$ has no vertices, we have $z_{a} \in W$. Further, vertex $z_{a}$ has degree $d+1$ in $G[W]$, and so reasoning as before, $T$ misses at least one vertex from the set $N\left(z_{a}\right)+z_{a}$. We conclude that, if $h=k d+d$, then $T$ has order at most $2 a d+2 b+2$.

Substituting $n=k+2+a(k d+d+1)+h$ and $h=b(k+1)+c$, in the left-hand side below, we obtain

$$
\left\lceil\frac{2 d n+2}{k d+d+1}\right\rceil=2 a d+2 b+2+\left\lceil\frac{2(d-b)+2 d c}{k d+d+1}\right\rceil \geqslant 2 a d+2 b+2,
$$

with the inequality following from the fact that $b \leqslant d$.

When $h=k d+d$, we showed that $T$ has order at most $2 a d+2 b+2$; thus, in this case, $|V(T)| \leqslant\lceil(2 d n+2) /(k d+d+1)\rceil$. That is, an optimal forest has at most this order for the infinitely many $n \geqslant k+2$ with $n \equiv k+1(\bmod k d+d+1)$. Together with the lower bound in Theorem 1, this shows that statement (ii) of the proposition holds.

We also showed that, if $c=0$, then $T$ has order at most $2 a d+b+3$. This shows that statement (i) holds when $c=0$. On the other hand, if $c \neq 0$, then $b<d$, and so the right-hand side of the above inequality can be increased to $2 a d+2 b+3$. Since we showed that the order of $T$ is always at most $2 a d+b+4$, we conclude that statement (i) of the proposition holds for all $n$.

By Proposition 9, we have the following.

Corollary 10. Let $k, d \geqslant 2$. Then the bound of Theorem 1 is tight to within 1 for all $n \geqslant 1$, and exactly sharp for infinitely many values of $n$.

In particular, the coefficient of $n$ in the bound of Theorem $1, r_{k, d}=2 d /(k d+d+1)$, is best possible.

\section{Other Values of $k$ and $d$}

We have established a lower bound on $f_{d}(G)$ when $k, d \geqslant 2$. Now we look at other values of $k, d$. We consider first $k<2$, and then $d<2$.

\subsection{Values of $k<2$}

If we set $k=0,1$, then the bound of Theorem 1 may not hold. In particular, for each $d \geqslant 2$ and $k=0,1$, there exist graphs for which the bound does not hold.

\subsection{1 $k=0$}

For $k=0$, this is obvious, since, when $d \geqslant 2$ and $k=0$, we have $r_{k, d}=2 d /(d+1)>1$. 
The actual bound when $k=0$ is trivial. The graphs of treewidth 0 are precisely the graphs with no edges. Thus, every induced subgraph of such a graph is a forest with maximum degree 0 , and so the correct bound, for $G$ a graph of order $n$, is given by

$$
f_{d}(G)=n .
$$

\subsection{2 $k=1$}

The case when $k=1$ is a bit more interesting. The graphs of treewidth at most 1 are precisely the forests. We seek a lower bound on the maximum order of an induced forest with bounded degree, in a forest.

First we show that, if we allow $k=1$, then the bound of Theorem 1 does not hold for $d \geqslant 2$. When $k=1$, we have $r_{k, d}=2 d /(2 d+1)$. Let $G=K_{1, d+1}$; this has order $n=d+2$. Then $f_{d}(G)=d+1=n \cdot(d+1) /(d+2)$, which is too small, since $(d+1) /(d+2)<2 d /(2 d+1)$ when $d \geqslant 2$.

This fraction $(d+1) /(d+2)$ gives us the best lower bound. The following result is due to Chappell, Gimbel, and Hartman [12]; it is restated using our notation. For completeness, we provide the proof (again, rewritten using our notation).

Proposition 11 (Chappell, Gimbel, \& Hartman). Let $d \geqslant 0$. If $G$ is a graph with treewidth at most 1 , (i.e., a forest) and order $n$, then

$$
f_{d}(G) \geqslant\left\lceil\frac{(d+1) n}{d+2}\right\rceil .
$$

Proof. We construct an induced forest $T$ of maximum degree at most $d$ and order at least $(d+1) n /(d+2)$. We proceed by induction on $n$. If $n \leqslant d+1$, then we may set $T=G$, and the result holds.

Suppose that $n \geqslant d+2$. If $G$ is not connected, then apply the induction hypothesis to each component.

We may thus assume that $G$ is a tree. Choose a vertex $x$ of $G$, and call $x$ the root of $G$, so that $G$ becomes a rooted tree, and we can talk about descendants in $G$. Consider the collection of all vertices of $G$ that have more than $d$ descendants. This collection is nonempty, since it contains the root. Let $v$ be a vertex in this collection that is as far from the root as possible.

Remove $v$ and all of its descendants from $G$, to obtain $G^{\prime}$. Apply the induction hypothesis to obtain a forest $T^{\prime}$, and let $T$ be the subgraph induced by the vertices in $T^{\prime}$, together with the descendants of $v$. The descendants of $v$ induce a subgraph with maximum degree at most $d$. Furthermore,

$$
\frac{|V(T)|-\left|V\left(T^{\prime}\right)\right|}{|V(G)|-\left|V\left(G^{\prime}\right)\right|} \geqslant \frac{d+1}{d+2},
$$

and so $T$ is the required forest. 
The example of $K_{1, d+1}$ shows that the fraction $(d+1) /(d+2)$ in Proposition 11 is best possible. Letting $G$ be a disjoint union of copies of $K_{1, d+1}$, plus one smaller star if $d+2$ does not divide $n$, we can verify that the bound of the proposition is sharp for all $n \geqslant 0$. If connected examples are desired, then add edges joining the various stars.

\subsection{Values of $d<2$}

\subsection{1 $d=0$}

When $d=0$, we seek a lower bound on the size of a maximum independent set in a graph $G$. If we allow $d=0$, then the bound of Theorem 1 is equal to 2 , since $r_{k, 0}=0$. This bound holds trivially, as long as $G$ is not a complete graph. However, we can find a better bound.

The best lower bound follows from the fact that a graph with treewidth at most $k$ is $(k+1)$-colorable. (To see this, find a chordal supergraph with clique number $k+1$, remove a simplicial vertex, color the remainder of the graph by induction, and then replace the simplicial, giving it a color not used on its neighbors, of which there are at most $k$.) Given such a coloring, we can take the largest color class. Thus, we obtain the following well known result. Note that $f_{0}(G)$ is the independence number of $G$.

Proposition 12. Let $k \geqslant 0$. If $G$ is a graph with order $n$ and treewidth at most $k$, then

$$
f_{0}(G) \geqslant\left\lceil\frac{n}{k+1}\right\rceil \text {. }
$$

Once again, the fraction $1 /(k+1)$ in Proposition 12 is best possible, and the proposition is sharp for all $n \geqslant 0$. To see this, let $G$ be a disjoint union of copies of $K_{k+1}$, plus one smaller clique if $k+1$ does not divide $n$. As before, we can add edges, if connected examples are desired.

\subsection{2 $d=1$}

The truly interesting case is that in which $d=1$.

If we allow $d=1$, then the bound of Theorem 1 does not hold. To see this, let $G=K_{k+2}-e$, that is, a complete graph on $k+2$ vertices with one edge removed. This $G$ has treewidth $k$, but the greatest order of an induced forest with maximum degree at most 1, is 2, which is too small. Larger counterexamples can be constructed using disjoint unions of copies of this graph; these may be joined by edges if connected counterexamples are desired.

However, although allowing $d=1$ would make the bound of Theorem 1 false, we suspect that it is "nearly true". It appears that the coefficient of $n$ (i.e., $r_{k, 1}$ ) is correct; the only problem is the " +2 " in the numerator. Thus, we make the following conjecture.

Conjecture 13. Let $k \geqslant 0$. If $G$ is a graph with order $n$ and treewidth at most $k$, then

$$
f_{1}(G) \geqslant\left\lceil\frac{2 n}{k+2}\right\rceil=\left\lceil r_{k, 1} \cdot n\right\rceil .
$$


If this conjecture holds, then the fraction $2 /(k+2)=r_{k, 1}$ is best possible. The bound would not be exactly sharp for all $n$; for example, when $k \geqslant 2$ and $n=2$, the above bound is 1 , while $f_{1}(G)=2$ for every 2 -vertex graph $G$. However, like Theorem 1 , the bound would be tight to within 1 for all $n$ and exactly sharp for infinitely many values of $n$. We can show this by letting $G$ be a disjoint union of copies of $K_{k+2}-e$, plus one smaller clique if $k+2$ does not divide $n$. And again, we can add edges, if connected examples are desired.

Later, we will prove Conjecture 13 for $k \leqslant 3$. However, although we cannot prove Conjecture 13 for all values of $k$, we can make use of Lemma 6 to prove the following weaker result for all $k \geqslant 2$.

Theorem 14. Let $k \geqslant 2$. If $G$ is a graph with order $n \geqslant 1$ and treewidth at most $k$, then

$$
f_{1}(G) \geqslant \frac{2 n+2}{2 k+3}
$$

Proof. Let $k, G$, and $n$ be as in the statement of the theorem. Define $G^{\prime}$ by attaching a partial vertex of weight $p=2 /(2 k-1)$ to each vertex of $G$, and let $P$ be the set of partial vertices. Note that our $p$ has the same value as that used in Lemma 6 when $d=2$ and $k$ is our given value. Apply Lemma 6 to $G^{\prime}$ with $k$ having the given value and $d=2$, to obtain $U^{\prime}$, and let $U=U^{\prime}-P$. Then $U$ induces a forest of maximum degree at most 1 in $G$. Also, $w\left(U^{\prime}\right) \geqslant r_{k, 2} \cdot w\left(G^{\prime}\right)+r_{k, 2} / 2$, with $w\left(G^{\prime}\right)=n+n p$, and so

$$
\begin{aligned}
w(U) & =w\left(U^{\prime}\right)-n p \\
& \geqslant r_{k, 2} \cdot w\left(G^{\prime}\right)+\frac{r_{k, 2}}{2}-n p \\
& =r_{k, 2} \cdot(n+n p)+\frac{r_{k, 2}}{2}-n p \\
& =\frac{4}{2 k+3}\left(n+\frac{2 n}{2 k-1}\right)+\frac{2}{2 k+3}-\frac{2 n}{2 k-1} \\
& =\frac{2 n+2}{2 k+3} .
\end{aligned}
$$

Again, we can prove the stronger bound of Conjecture 13 for $k \leqslant 3$.

Theorem 15. Let $k$ be an integer with $0 \leqslant k \leqslant 3$. If $G$ is a graph with order $n$ and treewidth at most $k$, then

$$
f_{1}(G) \geqslant\left\lceil\frac{2 n}{k+2}\right\rceil=\left\lceil r_{k, 1} \cdot n\right\rceil .
$$

The $k=0$ case of Theorem 15 follows from the discussion in Subsection 4.1.1. The $k=1$ case follows from Proposition 11. The $k=2$ and $k=3$ cases follow from the next two lemmas, respectively.

We prove the $k=2$ case using techniques similar to those used in the proof of Lemma 6 . However, the proof is much shorter, and there is no need for partial vertices. 
Lemma 16. If $G$ is a graph with order $n$ and treewidth at most 2 , then $G$ contains an induced forest $T$, so that $T$ has maximum degree at most 1 , and

$$
|V(T)| \geqslant \frac{2 n}{4}=r_{2,1} \cdot n
$$

Proof. Let $G$ and $n$ be as in the statement of the lemma. We proceed by induction on $n$. If $n=0$, then the result is trivially true; assume, therefore, that $n \geqslant 1$.

We may also assume that $G$ is connected and is either a 2-tree or a complete graph with at most 3 vertices. If not, then we can add edges to $G$, without increasing its treewidth beyond 2 , until it lies in one of these categories.

Let $G^{*}$ be the graph obtained from $G$ by removing every vertex that is simplicial in $G$.

If $V\left(G^{*}\right)=\varnothing$, that is, if every vertex in $G$ is simplicial, then $G$ is a complete graph with 1,2 , or 3 vertices. If $G$ is $K_{1}$ or $K_{2}$, then let $T=G$; if $G$ is $K_{3}$, then let $T$ be the subgraph induced by any 2 vertices of $G$, and we are done.

If there are two simplicial vertices $z$ and $z^{\prime}$ in $G$ that have the same (2-vertex) neighborhood, then delete this neighborhood, along with $z$ and $z^{\prime}$, to obtain $G^{\prime}$. Apply the induction hypothesis to obtain $T^{\prime}$, and let $T$ be $T^{\prime}+z+z^{\prime}$. Then

$$
\frac{|V(T)|-\left|V\left(T^{\prime}\right)\right|}{|V(G)|-\left|V\left(G^{\prime}\right)\right|}=\frac{2}{4} .
$$

Henceforth, we assume that $V\left(G^{*}\right) \neq \varnothing$, and that no two simplicial vertices in $G$ have the same neighborhood.

Depth 2 Cases - Let $y$ be a vertex that is simplicial in $G^{*}$. Let $X$ be the neighborhood of $y$ in $G^{*}$. Let $z_{1}, \ldots, z_{m}$ be the simplicial vertices in $G$ that are adjacent to $y$.

We claim that $|X|=2$. If $|X|<2$, then the removal of $X$ cannot disconnect $G$, because $G$ is a 2-tree, and so $X \cup\left\{y, z_{1}, \ldots, z_{m}\right\}$ is the entire vertex set of $G$. Further, there is exactly one possible neighborhood for the $z_{i}$ vertices: $X+y$, and so all of them have the same neighborhood. Thus, either there are 2 simplicials with the same neighborhood, or, if $m=1, G$ is a complete graph. Both of these cases have already been covered, and so we must have $|X|=2$. Say $X=\left\{x_{1}, x_{2}\right\}$.

There are exactly two possible neighborhoods for the simplicial vertices $z_{1}, \ldots, z_{m}$ : $\left\{x_{1}, y\right\}$ and $\left\{x_{2}, y\right\}$. Since no two simplicials can have the same neighborhood, we must have $m=1$ or $m=2$. (See Figure 13.)
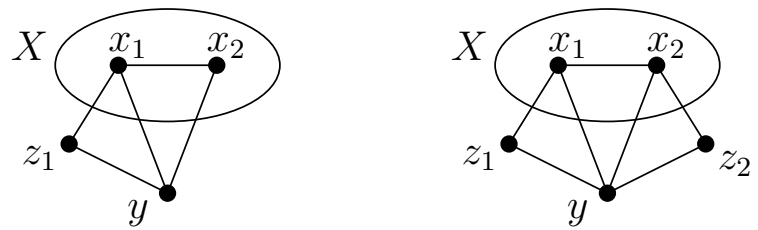

Figure 13: We must have $m=1$ or $m=2$ in the depth 2 cases of the proof of Lemma 16 . 
If $m=1$, then delete $X, y$, and $z_{1}$, to obtain $G^{\prime}$, apply the induction hypothesis to obtain $T^{\prime}$, and let $T$ be $T^{\prime}+y+z_{1}$. We have

$$
\frac{|V(T)|-\left|V\left(T^{\prime}\right)\right|}{|V(G)|-\left|V\left(G^{\prime}\right)\right|}=\frac{2}{4} .
$$

If $m=2$, then assume, without loss of generality, that each $z_{i}$ is adjacent to the corresponding $x_{i}$; that is, $N\left(z_{1}\right)=\left\{x_{1}, y\right\}$, and $N\left(z_{2}\right)=\left\{x_{2}, y\right\}$. Delete $z_{1}$ and $z_{2}$, to obtain $G^{\prime}$, and apply the induction hypothesis to obtain $T^{\prime}$.

We may assume that $y \in T^{\prime}$. If it is not, then we can add $y$ to $T^{\prime}$, removing one element of $X$ from $T^{\prime}$ if $T^{\prime}$ meets $X$, to obtain a new $T^{\prime}$ that contains $y$. Note that now $T^{\prime}$ contains at most 1 vertex of $X$.

Let $T=T^{\prime}-y+z_{1}+z_{2}$. Then

$$
\frac{|V(T)|-\left|V\left(T^{\prime}\right)\right|}{|V(G)|-\left|V\left(G^{\prime}\right)\right|}=\frac{2-1}{2}=\frac{2}{4} .
$$

This completes the proof of Lemma 16.

The remaining case of Theorem 15 is that in which $k=3$. For this case, we make use of partial vertices, as in Lemma 6 , along with a new kind of special vertex, which we call a "loner". A loner vertex is a simplicial vertex with degree at most 2 in $G$; if a loner lies in our induced forest, then no neighbor of this loner lies in the forest (thus the name). We denote the set of loner vertices by $Q$.

The $k=3$ case of Theorem 15 follows from the lemma below, by letting $P$ and $Q$ be empty.

Lemma 17. Let $G$ be a graph with order $n$ and treewidth at most 3. Let $P, Q$ be disjoint subsets of $V(G)$, so that each vertex in $P$ has degree at most 1 in $G$, each vertex in $Q$ is simplicial and has degree at most 2 in $G$, and $P \cup Q$ is an independent set in $G$. Let each vertex of $P$ have weight $1 / 3$, and let each other vertex of $G$, including those in $Q$, have weight 1 . Then there exists a set $U \subseteq V(G)$, such that all of the following hold.

(i) $G[U]$ is a forest of maximum degree at most 1.

(ii) $P \subseteq U$.

(iii) Each vertex in $Q \cap U$ has no neighbors in $G[U]$.

(iv) $w(U) \geqslant \frac{2}{5} w(G)=r_{3,1} \cdot w(G)$.

Proof. Let $G, n, P, Q$, and the vertex weights be as in the statement of the lemma. A vertex of weight $1 / 3$ is a partial vertex. A vertex having the restriction that, if it lies in $U$, then no neighbor lies in $U$, is a loner vertex. Thus, sets $P$ and $Q$ contain the partial and loner vertices of $G$, respectively. Partial and loner vertices are special; other vertices are full. 
We proceed by induction, first, on the weight of $G$, and second-for graphs of equal weight - on the number of full vertices.

Simple Cases-First, we deal with some simple base cases and reductions.

If some loner $u$ in $G$ has degree at most 1, then remove $u$ and its neighbor, if any, from $G$, to obtain $G^{\prime}$. Apply the induction hypothesis to obtain $U^{\prime}$, and let $U=U^{\prime}+u$. Then

$$
\frac{w(U)-w\left(U^{\prime}\right)}{w(G)-w\left(G^{\prime}\right)} \geqslant \frac{1}{2}>\frac{2}{5}
$$

If 2 special vertices have intersecting neighborhoods, then delete these specials and their neighborhoods (of 1, 2, or 3 full vertices total), to obtain $G^{\prime}$. (See Figure 14 for possibilities.) Apply the induction hypothesis to obtain $U^{\prime}$, and let $U$ be $U^{\prime}$ plus the 2 deleted specials. If both specials are partial, then the fraction $\left[w(U)-w\left(U^{\prime}\right)\right] /[w(G)-$ $\left.w\left(G^{\prime}\right)\right]$ is $(2 / 3) /(1+2 / 3)=2 / 5$. If one is a partial vertex and one is a loner, then the fraction is $(1 / 3+1) /(2+1 / 3+1)=2 / 5$. If both specials are loners, then the fraction is $2 / 5$ if the neighborhoods have exactly 1 vertex in common, and $2 / 4$ if they have 2 vertices in common - at least $2 / 5$ in either case.
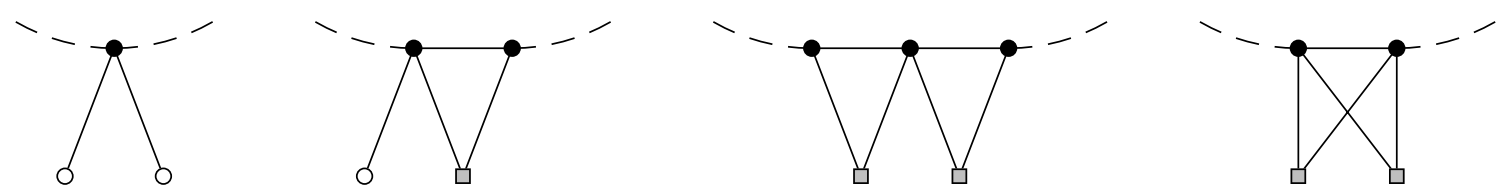

Figure 14: Special vertices with intersecting neighborhoods. Loners are gray squares.

Henceforth, we assume that each loner in $G$ has degree 2, and that no full vertex is adjacent to more than 1 special.

Suppose that there are at most 3 full vertices. If there are no full vertices, then let $U=V(G)$. If there are no special vertices, then let $U$ contain $\min \{2, n\}$ vertices of $G$. If there are partial vertices but no loners, then let $U$ be all the partial vertices, together with 1 full vertex. Otherwise, there is a loner, and so there are either 2 or 3 full vertices, and possibly 1 partial vertex if there are 3 full vertices. Let $U$ be the two neighbors of the loner, plus the partial vertex, if there is one. In each case, $U$ has the proper structure and at least the required weight.

Henceforth, we assume that $G$ has at least 4 full vertices. Further, we may assume that $G$ is connected and $G-P-Q$ is a 3-tree; otherwise, add edges until these are true.

A Simplicial Vertex-Now consider a vertex $z$ that is simplicial in $G-P-Q$. Let $X=\left\{x_{1}, x_{2}, x_{3}\right\}$ be the neighborhood of $z$ in $G-P-Q$.

Suppose that $z$ has a loner neighbor $u$. Without loss of generality, say the other neighbor of $u$ is $x_{1}$. Remove $z$ and $u$ and add a new partial vertex $c$ adjacent to $x_{1}$, to obtain $G^{\prime}$. Apply the induction hypothesis to obtain $U^{\prime}$. If $x_{1} \notin U^{\prime}$, then we may let 

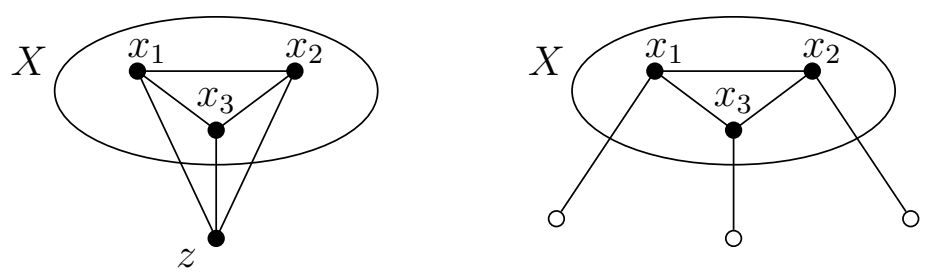

Figure 15: When a simplicial vertex $z$ is not adjacent to any special (before and after).

$U=U^{\prime}-c+u$. If $x_{1} \in U^{\prime}$, then, noting that $c \in U^{\prime}$, we see that $x_{2}$ and $x_{3}$ cannot lie in $U^{\prime}$; let $U=U^{\prime}-c+z$. In either case,

$$
\frac{w(U)-w\left(U^{\prime}\right)}{w(G)-w\left(G^{\prime}\right)}=\frac{1-\frac{1}{3}}{2-\frac{1}{3}}=\frac{2}{5} .
$$

Now suppose that $z$ is not adjacent to any special. Remove $z$ and add three partial vertices, one adjacent to each element of $X$, to obtain $G^{\prime}$. (See Figure 15.) This does not change the weight of the graph, but it does reduce the number of full vertices. Apply the induction hypothesis to obtain $U^{\prime}$. Note that $\left|X \cap U^{\prime}\right| \leqslant 1$. Let $U$ be $U^{\prime}+z$ minus the three added partial vertices. Since $w(G)=w\left(G^{\prime}\right)$ and $w(U)=w\left(U^{\prime}\right)$, the weight requirement is satisfied.

Henceforth, we assume that each vertex that is simplicial in $G-P-Q$ is adjacent to exactly one partial vertex and no loners.

If there is a vertex $z^{\prime} \neq z$ that is simplicial in $G-P-Q$ and whose neighborhood in $G-P-Q$ is $X$ (the neighborhood of $z$ ), then remove $X, z, z^{\prime}$, and their 2 partial neighbors, to obtain $G^{\prime}$. Apply the induction hypothesis to obtain $U^{\prime}$, and let $U$ be $U^{\prime}$ plus $z, z^{\prime}$, and their 2 partial neighbors. Then

$$
\frac{w(U)-w\left(U^{\prime}\right)}{w(G)-w\left(G^{\prime}\right)}=\frac{2+\frac{2}{3}}{5+\frac{2}{3}}>\frac{2}{5} .
$$

Henceforth, we assume that no two vertices that are simplicial in $G-P-Q$ have the same neighborhood in $G-P-Q$.

Suppose there is a loner $u$ adjacent to an element of $X$; say $N(u)=\left\{x_{1}, v\right\}$, where $v$ may or may not lie in $X$. (See Figure 16.) Remove $u, x_{1}, v$, and the partial vertex adjacent to $z$, and make $z$ into a loner, to obtain $G^{\prime}$. Apply the induction hypothesis to obtain $U^{\prime}$, and let $U$ be $U^{\prime}$ plus $u$ and the partial vertex adjacent to $z$. Then

$$
\frac{w(U)-w\left(U^{\prime}\right)}{w(G)-w\left(G^{\prime}\right)}=\frac{1+\frac{1}{3}}{3+\frac{1}{3}}=\frac{2}{5} .
$$

Henceforth, we assume that no loner is adjacent to any neighbor of a vertex that is simplicial in $G-P-Q$. 

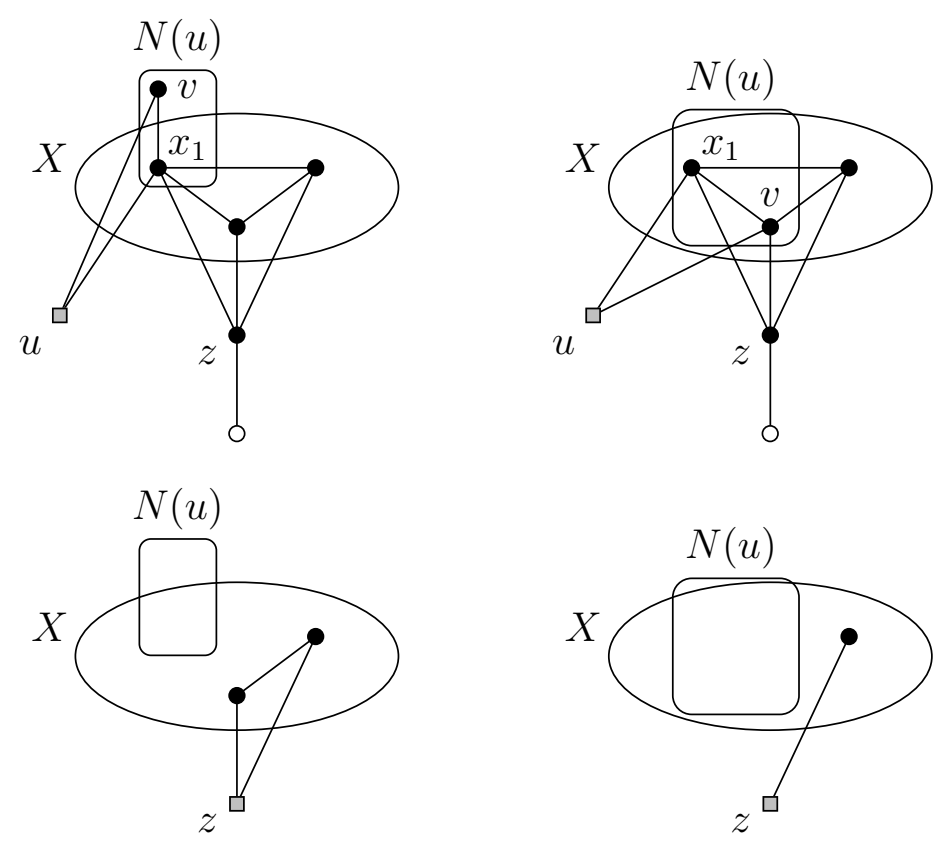

Figure 16: When there is a loner adjacent to $X$ (both possibilities, before and after).

The Subgraph $G^{*}$ - Let $G^{*}$ be the graph obtained from $G-P-Q$ by removing each vertex that is simplicial in $G-P-Q$.

If $V\left(G^{*}\right)=\varnothing$, that is, if every vertex of $G-P-Q$ is simplicial in $G-P-Q$, then $G$ is a complete graph on 4 full vertices with a partial vertex joined to each full vertex. Let $U$ be all the partial vertices, together with one full vertex. Then

$$
\frac{w(U)}{w(G)}=\frac{1+\frac{4}{3}}{4+\frac{4}{3}}>\frac{2}{5} .
$$

Henceforth, we assume that $V\left(G^{*}\right) \neq \varnothing$, and thus that $G-P-Q$ is not a complete graph. Since $G-P-Q$ is a 3 -tree, it must contain two nonadjacent simplicial vertices. Two simplicial vertices of $G-P-Q$ cannot have the same neighborhood, and so $\left|V\left(G^{*}\right)\right|>3$. Thus, $G^{*}$ is a 3 -tree.

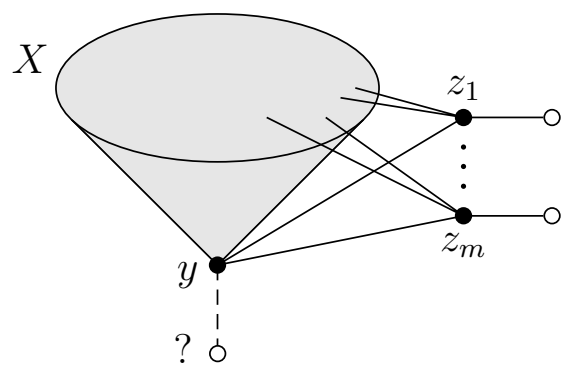

Figure 17: Depth 2 Cases. 
Depth 2 Cases - Let $y$ be a vertex that is simplicial in $G^{*}$. Let $X$ be the neighborhood of $y$ in $G^{*}$. Since $G^{*}$ is a 3 -tree, we must have $|X|=3$. Let $z_{1}, \ldots, z_{m}$ be the full simplicial vertices in $G$ that are adjacent to $y$. Then each $z_{i}$ is adjacent to exactly one partial vertex, $y$ may or may not be adjacent to a single partial vertex, and no loner is adjacent to $y$ or to any of the $z_{i}$ vertices. (See Figure 17.)

If $m \geqslant 2$, then remove $X, y$ and the $z_{i}$ vertices, and their adjacent partial vertices, to obtain $G^{\prime}$. Apply the induction hypothesis to obtain $U^{\prime}$, and let $U$ be $U^{\prime}$ plus the $z_{i}$ vertices and their adjacent partial vertices, along with the partial vertex adjacent to $y$, if there is one. Then

$$
\begin{aligned}
\frac{w(U)-w\left(U^{\prime}\right)}{w(G)-w\left(G^{\prime}\right)} & \geqslant \frac{m+m \cdot \frac{1}{3}}{4+m+m \cdot \frac{1}{3}} & & \text { minimized when } y \text { has no adjacent partial vertex } \\
& \geqslant \frac{2+\frac{2}{3}}{6+\frac{2}{3}}=\frac{2}{5} . & & \text { minimized when } m=2
\end{aligned}
$$

It remains to prove the result when $m=1$. Let $x_{1}, x_{2}$ be the neighbors of $z_{1}$ in $X$. (See Figure 18.)
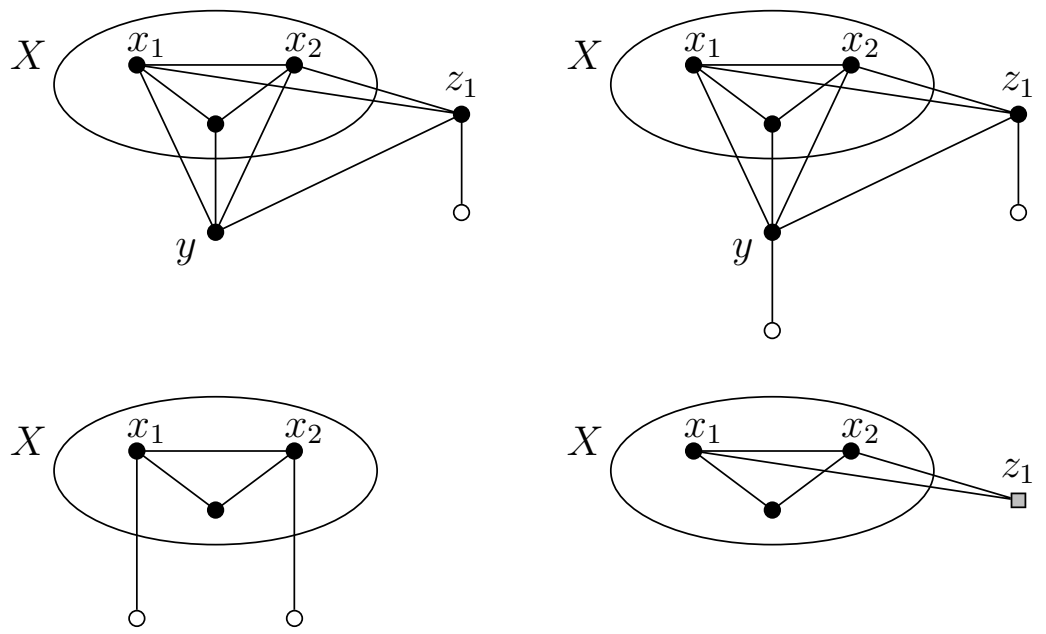

Figure 18: When $m=1$ and $y$ is not (left) or is (right) adjacent to a partial vertex (before and after).

If $y$ is not adjacent to a partial vertex, then remove $z_{1}$, its neighboring partial vertex, and $y$, and then add two new partial vertices adjacent to $x_{1}, x_{2}$, respectively, to obtain $G^{\prime}$. Apply the induction hypothesis to obtain $U^{\prime}$. At most one vertex of $X$ will lie in $U^{\prime}$. If $x_{1}$ or $x_{2}$ lies in $U^{\prime}$, then let $U$ be $U^{\prime}$ minus the two added partial vertices, plus $y$ and the removed partial vertex. Otherwise, let $U$ be $U^{\prime}$ minus the two added partial vertices, plus $z_{1}$ and the removed partial vertex. In either case,

$$
\frac{w(U)-w\left(U^{\prime}\right)}{w(G)-w\left(G^{\prime}\right)}=\frac{-\frac{2}{3}+1+\frac{1}{3}}{-\frac{2}{3}+2+\frac{1}{3}}=\frac{2}{5} .
$$


If, on the other hand, $y$ is adjacent to a partial vertex, then remove $y$ and the partial vertices adjacent to $y$ and $z_{1}$, and make $z_{1}$ a loner, to obtain $G^{\prime}$. Apply the induction hypothesis to obtain $U^{\prime}$, and let $U$ be $U^{\prime}$ plus the removed partial vertices. Then

$$
\frac{w(U)-w\left(U^{\prime}\right)}{w(G)-w\left(G^{\prime}\right)}=\frac{\frac{2}{3}}{1+\frac{2}{3}}=\frac{2}{5} .
$$

This completes the proof of Lemma 17 .

We have now proven Theorem 15 .

\section{Graphs on Surfaces}

This research was initially motivated by two conjectures of the first author. The first conjecture stated that, if $G$ is an outerplanar graph of order $n$, then $f_{2}(G)>4 n / 7$. This conjecture was proven by the second author [21, Thm. 1]. Because outerplanar graphs have treewidth at most 2 , and $r_{2,2}=4 / 7$, this result is implied by our more general Theorem 1.

The second conjecture is a similar one involving planar graphs. It remains open.

Conjecture 18. Let $G$ be a planar graph of order $n \geqslant 1$. Then $f_{2}(G)>4 n / 9$.

While we do not know whether the above conjecture holds, we do know that, if it does, then the fraction $4 / 9$ is best possible, because of the following observation.

Observation 19. If $k=3$, then, for all $n \geqslant 1$ and $d \geqslant 2$, the graph $G(k, d, n)$, from Example 8, is planar.

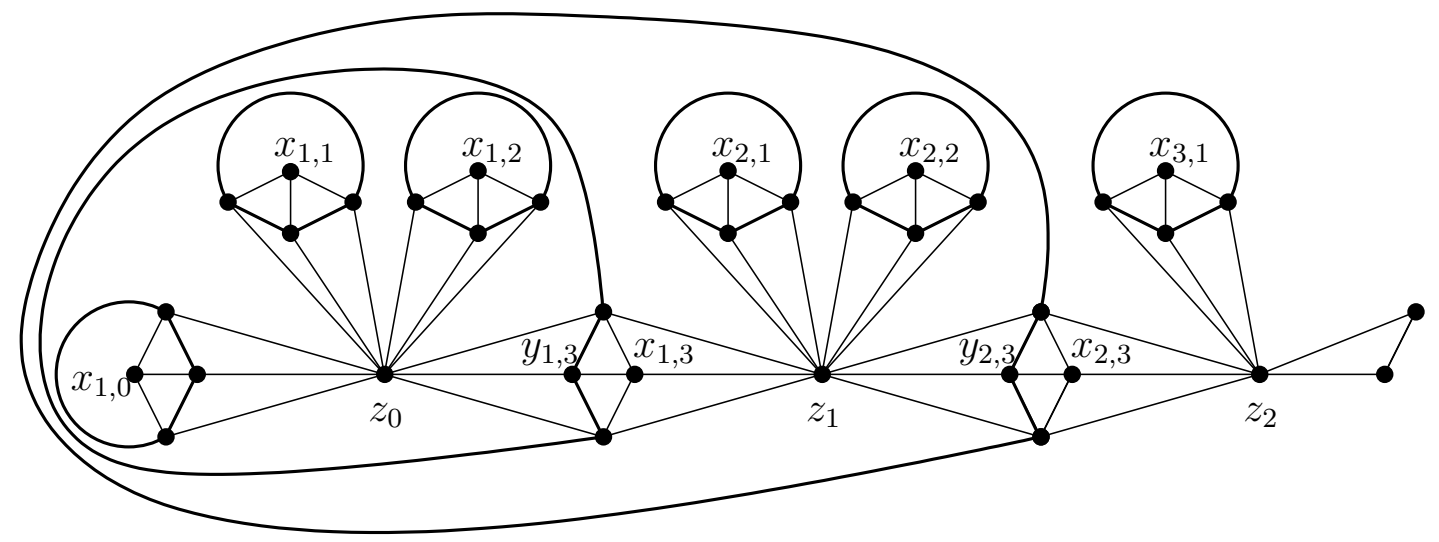

Figure 19: $G(k, d, n)$ with $n=37, k=3, d=3$ (thus $a=2, h=6, b=1$, and $c=2$ ), drawn without crossing edges to illustrate the fact that it is planar. Edges within each $S_{i, j}$ are shown in bold. 
(See Figure 19 for an illustration.) By this observation, we can use the graphs $G(3, d, n)$, along with Proposition 9, to prove upper bounds for planar graphs.

A weaker version of Conjecture 18, in which " $f_{2}(G)>4 n / 9$ " is replaced by " $f_{2}(G) \geqslant$ $n / 3$ ", is known to hold. This follows from a result proved independently by Poh [22] and by Goddard [16]: that every planar graph has a (not necessarily proper) vertex 3-coloring, so that each color class induces a forest of maximum degree at most 2 (a linear forest). In such a coloring, the largest color class induces a linear forest with at least $n / 3$ vertices.

While planar graphs can have arbitrarily high treewidth (this is well known, but nontrivial to prove; see, for example, Diestel [13]), in many ways they are similar to graphs with treewidth at most 3 . For example, the maximum number of edges in an $n$-vertex planar graph is $3 n-6$; the same holds for $n$-vertex graphs of treewidth at most 3. Both classes have a maximum clique number of 4 ; indeed, the maximum order of a clique minor is 4 . Our investigations suggest that planar graphs also behave much like graphs of treewidth at most 3 for the purpose of finding large induced forests. If $k=3$, then $r_{k, d}=2 d /(4 d+1)$. We conjecture that this same fraction gives a lower bound for planar graphs.

Conjecture 20. Let $d \geqslant 2$ be an integer. If $G$ is a planar graph with order $n \geqslant 1$, then

$$
f_{d}(G)>\frac{2 d n}{4 d+1}=r_{3, d} \cdot n .
$$

Note that Conjecture 20 generalizes Conjecture 18, since $2 d /(4 d+1)=4 / 9$ when $d=2$; that is, $r_{3,2}=4 / 9$. And as before, if the above conjecture is true, then the fraction $2 d /(4 d+1)$ is best possible, as shown by the graphs $G(k, d, n)$ from Example 8 , with $k=3$.

Conjecture 20 would become false if we allowed $d=1$, as shown by setting $G=K_{2,2,2}$ (the octahedron). The largest induced forest with maximum degree at most 1 in this graph has 2 vertices, while, when $d=1$ and $n=6$, we have $2 d n /(4 d+1)=12 / 5>2$.

Letting $d$ increase without bound, the value of $r_{3, d}$ approaches $1 / 2$. Thus, Conjecture 20 implies the Induced Forest Conjecture of Albertson and Berman [2]: that every $n$-vertex planar graph contains an induced forest with at least $n / 2$ vertices.

If " $n / 2$ " is replaced by " $2 n / 5$ ", then the resulting statement is known to hold. This follows from a result of Borodin [8, 9]: that every planar graph has a proper vertex 5coloring, so that the union of each pair of color classes induces a forest. In such a coloring, the union of the two largest color classes induces a forest with at least $2 n / 5$ vertices.

Note that proving only Conjecture 18 would not suffice to verify the Induced Forest Conjecture. However this would still improve on the $2 n / 5$ in the previous paragraph, since $4 / 9>2 / 5$.

Lastly, we consider induced forests in graphs on other surfaces. We ask whether the ratio $2 d /(4 d+1)$ works for any surface, once a correction term is subtracted.

Question 21. Does there exist a function $\varphi$ so that, if $d \geqslant 2$ is an integer and $G$ is a graph of order $n$ that is embeddable on a surface $S$, then

$$
f_{d}(G) \geqslant \frac{2 d}{4 d+1} \cdot[n-\varphi(S)] ?
$$


In particular, when $S$ is the torus, does $\varphi(S)=3$ work?

This idea of being able to remove a fixed number of vertices from a graph embeddable on some surface, in order to obtain a graph that "acts planar", is not a new one. In particular, Albertson [1] conjectured that for each surface $S$ there is a constant $q(S)$, so that for each graph $G$ embeddable on $S$, a set of at most $q(S)$ vertices can be removed from $G$, to obtain a 4 -colorable graph.

\section{Acknowledgements}

The authors would like to thank John Gimbel and Chris Hartman for allowing us to include their result.

\section{References}

[1] M. O. Albertson. Open problem 2. In The Theory and Applications of Graphs (G. Chartrand et al., eds.), page 609. Wiley, 1981.

[2] M. O. Albertson and D. Berman. A conjecture on planar graphs. In Graph Theory and Related Topics (J. A. Bondy and U. S. R. Murty, eds.), page 357. Academic Press, 1979.

[3] M. Albertson, B. Bollobás, and S. Tucker. The independence ratio and maximum degree of a graph. In Proceedings of the Seventh Southeastern Conference on Combinatorics, Graph Theory and Computing (Louisiana State Univ., Baton Rouge, La., 1976), volume XVII of Congress. Numer., pages 43-50. Utilitas Mathematica, Winnipeg, Man., 1976.

[4] N. Alon, D. Mubayi, and R. Thomas. Large induced forests in sparse graphs. J. Graph Theory, 38(3):113-123, 2001.

[5] J. Akiyama and M. Watanabe. Maximum induced forests of planar graphs, Graphs Combin., 3(1):201-202, 1987.

[6] S. Bau and L. W. Beineke. The decycling number of graphs, Australas. J. Combin., 25:285-298, 2002.

[7] H. L. Bodlaender. A partial $k$-arboretum of graphs with bounded treewidth. Theoret. Comput. Sci., 209(1-2):1-45, 1998.

[8] O. V. Borodin. A proof of B. Grünbaum's conjecture on the acyclic 5-colorability of planar graphs (Russian). Dokl. Akad. Nauk SSSR, 231(1):18-20, 1976.

[9] O. V. Borodin. On acyclic colorings of planar graphs. Discrete Math., 25(3):211-236, 1979.

[10] P. Bose, V. Dujmović, and D. R. Wood. Induced subgraphs of bounded degree and bounded treewidth. Contrib. Discrete Math., 1(1):88-105, 2006.

[11] I. Caragiannis, C. Kaklamanis, and P. Kanellopoulos. New bounds on the size of the minimum feedback vertex set in meshes and butterflies. Inform. Process. Lett., 83(5):275-280, 2002. 
[12] G. G. Chappell, J. Gimbel, and C. Hartman. Generalized independence in graphs. Manuscript.

[13] R. Diestel. Graph theory, 2nd ed. Graduate Texts in Mathematics, 173. SpringerVerlag, New York, 2000.

[14] S. Fajtlowicz. On the size of independent sets in graphs, In Proceedings of the Ninth Southeastern Conference on Combinatorics, Graph Theory, and Computing (Florida Atlantic Univ., Boca Raton, Fla., 1978), volume XXI of Congress. Numer., pages 269-274. Utilitas Mathematica, Winnipeg, Man., 1978.

[15] G. Fertin, E. Godard, and A. Raspaud. Minimum feedback vertex set and acyclic coloring. Inform. Process. Lett. 84(3):131-139, 2002.

[16] W. Goddard. Acyclic colorings of planar graphs. Discrete Math., 91(1):91-94, 1991.

[17] C. C. Heckman and R. Thomas. Independent sets in triangle-free cubic planar graphs. J. Comb. Theory Ser. B, 96(2):253-275, 2006.

[18] K. Hosono. Induced forests in trees and outerplanar graphs. Proc. Fac. Sci. Tokai Univ., 25:27-29, 1990.

[19] K. F. Jones. Size and independendence in triangle-free graphs with maximum degree three. J. Graph Theory, 14(5):525-535, 1990.

[20] S. C. Locke and F. Lou. Finding independent sets in $K_{4}$-free 4-regular connected graphs. J. Combin. Theory Ser. B, 71(1):85-110, 1997.

[21] M. J. Pelsmajer. Maximum induced linear forests in outerplanar graphs. Graphs Combin., 20(1):121-129, 2004.

[22] K. S. Poh. On the linear vertex-arboricity of a planar graph. J. Graph Theory, 14(1):73-75, 1990.

[23] B. A. Reed. Tree width and tangles: a new connectivity measure and some applications. In Surveys in combinatorics, 1997 (London), volume 241 of London Math. Soc. Lecture Note Ser., pages 87-162. Cambridge Univ. Press, Cambridge, 1997.

[24] M. R. Salavatipour. Large induced forests in triangle-free planar graphs. Graphs Combin., 22(1):113-126, 2006.

[25] W. Staton. Some Ramsey-type numbers and the independence ratio. Trans. Amer. Math. Soc., 256:353-370, 1979.

[26] D. B. West. Introduction to Graph Theory, 2nd ed. Prentice-Hall, Upper Saddle River, NJ, 2001. 